\title{
Weak decay of hypernuclei
}

\author{
A. Parreño and A. Ramos \\ Departament d'Estructura i Constituents de la Matèria, Facultat de Física, Diagonal 647, E-08028 Barcelona, Spain \\ C. Bennhold \\ Center of Nuclear Studies, Department of Physics, The George Washington University, Washington, DC 20052
}

(Received 15 November 1996)

\begin{abstract}
The nonmesonic weak decay of $\Lambda$ hypernuclei is studied in a shell model framework. A complete strangeness-changing weak $\Lambda N \rightarrow N N$ transition potential, based on one boson exchange, is constructed by including the exchange of the pseudoscalar mesons $\pi, K, \eta$ as well as the vector mesons $\rho, \omega$, and $K^{*}$, whose weak-coupling constants are obtained from soft meson theorems and SU(6) ${ }_{w}$. General expressions for nucleons in arbitrary shells are obtained. The transition matrix elements include realistic $\Lambda N$ short-range correlations and $N N$ final state interactions based on the Nijmegen baryon-baryon potential. The decay rates are found to be especially sensitive to the inclusion of the strange mesons $K$ and $K^{*}$ even though the role of kaon exchange is found to be reduced with recent couplings obtained from one-loop corrections to the leading order in chiral perturbation theory. With the weak couplings used in this study the rates remain dominated by the pion-exchange mechanism since the contributions of heavier mesons either cancel each other or are suppressed by form factors and short-range correlations. The total decay rate therefore remains in agreement with present measurements. However, the partial rates which are even more sensitive to the inclusion of heavier mesons cannot be reconciled with the data. The proton asymmetry changes by $50 \%$ once heavier mesons are included and agrees with the available data. [S0556-2813(97)05807-X]
\end{abstract}

PACS number(s): 21.80.+a, 13.75.Ev, 25.80.Pw

\section{INTRODUCTION}

In single $\Lambda$ hypernuclei, a $\Lambda$ hyperon can occupy any orbital in the hypernucleus since it is free from the Pauli exclusion principle due to its additional quantum number strangeness. Hypernuclei are typically produced in some excited state through hadronic reactions such $\left(K^{-}, \pi^{-}\right)$or $\left(\pi^{+}, K^{+}\right)$but can reach their ground state through electromagnetic $\gamma$ and/or nucleon emission. Eventually, they will decay through weak interaction processes which involve the emission of pions or nucleons but are nonleptonic in nature.

A free $\Lambda$ hyperon has a lifetime of about 260 ps and decays almost totally into a pion and a nucleon $\left[\Lambda \rightarrow p \pi^{-}\right.$ $\left.(\sim 64 \%), \Lambda \rightarrow n \pi^{0}(\sim 36 \%)\right]$, with a release of kinetic energy of about $5 \mathrm{MeV}$ to the nucleon along with a corresponding final momentum of about $100 \mathrm{MeV} / c$. When the $\Lambda$ is embedded in the nuclear medium, the phase space for the mesonic decay is greatly reduced since the $\Lambda$ is bound by some $10 \mathrm{MeV}$ for $p$-shell hypernuclei and up to $30 \mathrm{MeV}$ in heavy hypernuclei. The final-state nucleon with its very low momentum thus becomes Pauli blocked, leading to a suppression of the mesonic rate by several orders of magnitude for heavy hypernuclei such as ${ }_{\Lambda}^{208} \mathrm{~Pb}$. Experimentally, however, one finds the lifetimes of hypernuclei to be roughly independent of $A$ (see, e.g., Fig. 14-1 in Ref. [1]), though the data base is very poor, especially for systems with $A>12$. Therefore, the nuclear medium surrounding the bound $\Lambda$ affects its weak decay by introducing new, nonmesonic decay modes, such as $\Lambda N \rightarrow N N$. Thus, hypernuclei larger than ${ }_{\Lambda}^{5} \mathrm{He}$ decay mainly through these nonmesonic channels, where the $\Lambda$ mass excess of $176 \mathrm{MeV}$ is converted into kinetic energy of a final state of nucleons emerging with a momentum of about $400 \mathrm{MeV} / c$. As a result, this process has a much larger phase space relative to the mesonic one, and the outgoing nucleons are not Pauli blocked.

This was recognized more than 40 years ago [2] when it was suggested that the novel two-baryon decay mode $\Lambda N \rightarrow N N$ could be understood in terms of the free-space decay mechanism $\Lambda \rightarrow \pi N$ with the exception that the pion now has to be considered virtual and is absorbed on a second nucleon bound in the hypernucleus. However, the large momentum transfer involved in the reaction leads to a mechanism that is sensitive to the short-distance behavior of the amplitude and thus raises the possibility that the exchange of heavier mesons may play an important role. The production of these mesons would be below threshold for the free-space $\Lambda$ decay, but they can contribute through virtual exchange in a two-baryon decay channel. As discussed further below it is the kinematic freedom of these additional boson exchanges that provides part of the motivation for this study. The fairly large momentum transfer also raises the hope that this reaction turns out to be insensitive to nuclear structure details and thus creates a suitable channel to investigate the weak decay mechanism.

We would like to point out that there is another possible nonmesonic decay channel, the two-nucleon induced process $\Lambda N N \rightarrow N N N$, where the virtual pion emitted at the weak vertex is absorbed by a pair of nucleons which are correlated through the strong force. This mechanism was first investigated in Ref. [3] where it was suggested that its magnitude could be comparable to $\Lambda N \rightarrow N N$. However, a reanalysis with more realistic assumptions [4,5] reduced its contribution to $10-15 \%$ of the total nonmesonic decay rate. Its relevance lies mainly in its potential to renormalize the experi- 
mentally measured partial ratios; we will return to this point later when discussing results.

The main goal in studying the weak nonmesonic decay channel is to gain insight into the fundamental aspects of the four-fermion, strangeness changing weak interaction. Most of the earlier work on nonleptonic weak processes proceeded directly from some model weak Hamiltonian and computed experimental observables that could then be compared to measurements. More recently the approach has been divided into a two-stage process. The first step starts with the standard model electroweak Hamiltonian at the subhadronic level and takes into account QCD corrections at short distances, yielding a so-called effective weak Hamiltonian. In relation to the mass of the $W$ boson of $80 \mathrm{GeV}$, these would be low momentum transfer processes leading to a zero-range or contact interaction. The Cabbibo theory combined with strong-interaction corrections would result in $V-A$ weak interaction and presumably predict the relative strength of the $\Delta S=0$ and $\Delta S=1$ transition. Thus, hadronic weak matrix elements of the form $\left\langle M B^{\prime}\left|H_{w}\right| B\right\rangle$ could eventually be calculated. The second stage involves using these weak vertices as a starting point for effective nuclear two-body operators that are then implanted into the nucleus with the usual nuclear many-body wave functions. It is the latter task which is the main subject of this paper.

The most important information regarding the nonleptonic weak interactions of hyperons comes from their free-space decays. However, while these processes have been wellmeasured and understood phenomenologically, a more basic understanding in terms of the underlying degrees of freedom is still lacking. The situation is similar for the nonmesonic decay modes, except that the experimental data are more unsatisfactory. The early measurements were based on bubble chamber experiments and emulsion works, who suffered from low precision, poor statistics, and difficulties with the identification of the particular hypernucleus, except for the very light hyperfragments. These experiments were nevertheless able to establish the first limits on hypernuclear lifetimes, albeit with large uncertainties. Their techniques were mostly aimed at measuring the emission of the $\pi^{-}$ from the mesonic decay channel; the analyses of the nonmesonic decay modes were greatly inhibited by the low hypernuclear production rates, the limited spacial resolution of early detectors and the presence of one or more neutral particles (especially neutrons) in the final state. In recent years a series of counter experiments carried out at BNL and KEK improved the quality of data on the nonmesonic decay modes using pion and kaon beams. In contrast to these measurements LEAR at CERN explored hypernuclei in the $A=200$ mass region following antiproton annihilation [6]. The heavy hypernuclei were identified through their delayed fission events which were attributed to the nonmesonic decays. While this interpretation is fraud with difficulties it resulted in lifetimes similar to the those of $p$-shell hypernuclei, thus raising the intruiging possibility that the $\Lambda N \rightarrow N N$ mechanism saturates already in the $A=12$ system. On the other hand, employing the $\left(K^{-}, \pi^{-}\right)$production mechanism with direct timing techniqes, the Brookhaven AGS measurements resulted in better data on the hypernuclear lifetimes and branching ratios of mesonic as well as nonmesonic decay channels of ${ }_{\Lambda}^{12} \mathrm{C},{ }_{\Lambda}^{11} \mathrm{~B}$ and the different helium hypernuclei
$[7,8]$. The group at KEK used the $\left(\pi^{+}, K^{+}\right)$production reaction to not only measure total and partial decay rates [9], but also to use the induced polarization in ${ }_{\Lambda}^{12} \mathrm{C}$ to determine for the first time the difference in the number of protons emitted along the axis of polarization compared to the number ejected in the opposite direction [10]. This asymmetry is a direct consequence of the presence of parity-violating components in the weak decay mechanism.

Parity violation in hadronic systems represents a unique tool to study aspects of the nonleptonic weak interaction between hadrons. The nonmesonic process resembles the weak $\Delta S=0$ nucleon-nucleon interaction that has been explored experimentally in parity-violating $N N$ scattering measurements by measuring the asymmetry of longitudinally polarized protons. However, the $\Lambda N \rightarrow N N$ two-body decay mode contains more information since it can explore both the parity-conserving (PC) and the parity-violating (PV) sector of the $\Delta S=1$ weak baryon-baryon interaction while in the weak $N N$ system the strong force masks the signal of the weak PC interaction. On the other hand, the free process $\Lambda N \rightarrow N N$ cannot be accessed experimentally which complicates the interpretation of the nonmesonic decay rates since the reaction mechanism has to be studied in the environment of hypernuclear structure.

A number of theoretical approaches to the $\Lambda N \rightarrow N N$ decay mode have been developed over the last thirty years which are more extensively reviewed in Ref. [11]. The early phenomenological analyses by Dalitz et al. [12] provided the general nonrelativistic structure of the $\Lambda N \rightarrow N N$ amplitude which was then related to decay rates of $s$-shell hypernuclei using certain simplifying assumptions. The $\Delta S=0$ weak nucleon-nucleon interaction at low and intermediate energies has generally been described in a meson exchange model involving one strong interaction vertex and one weak one; the same basic assumption has been used for a microscopic description of the $\Delta S=1 \Lambda N \rightarrow N N$ mechanism. Early calculations based on the one-pion exchange (OPE) were due to Adams [13], modifications of the OPE due to strong interactions in the nuclear medium were suggested in Ref. [14] to account for many-body nuclear structure effects. At the very least, the OPE mechanism can be expected to adequately descibe the long-range part of the $\Lambda N \rightarrow N N$ interaction. The first attempts to include heavier bosons, at first the $\rho$ meson - again in complete analogy to the $\Delta S=0 N N$ interactionwere presented in Refs. $[15,16]$. There were several conference papers by Dubach et al. [17] showing results of preliminary calculations with a full meson exchange potential; a more detailed account of their calculations has recently become available [18]. Finally, for completeness we mention that a study of $\Lambda N \rightarrow N N$ based on quark rather than meson degrees was carried out in Ref. [20]. Their model separates the process into a long-range region, to be described by OPE, and a short-range region, modeled by a six-quark interaction with suitably adjusted parameters. This idea was revived in Ref. [21]. Results for light hypernuclei showed that the total rates preferred a relative plus sign between quark and OPE contributions, while the neutron to proton ratio was better reproduced with a minus sign. In the calculation of Ref. [22] both OPE and OKE (one-kaon exchange) amplitudes were considered, in addition the the quark ones, and significant cancellations between OPE and OKE contributions were 
found. Finally, there have been some attempts to incorporate the exchange of the $\sigma$ and $\rho$ mesons from the point of view of a correlated two-pion exchange $[23,24]$, where the weak vertex was obtained through the coupling of the two pions to the $\sigma$ or $\rho$ and intermediate $N$ and $\Sigma$ baryon states. Both calculations find an enhancement of the central transitions leading to a moderate increase of the neutron to proton ratio.

The motivation for this work is twofold. First, in contrast to most previous investigations performed in nuclear matter this study analyses the nonmesonic hypernuclear decay in a shell model framework. Spectroscopic factors are employed to describe the initial hypernuclear and final nuclear structure as well as possible. Rather than constraining the $\Lambda N$ system to only $l=0$, all possible initial and final relative orbital angular momenta are included. To reduce the uncertainties regarding initial and final short-range correlations (SRC) we use realistic $\Lambda N$ and $N N$ interactions based on the Nijmegen baryon-baryon potential. The nuclear structure details are thus treated with as few approximations and ambiguities as possible. We emphasize here that our current treatment is nonrelativistic, in contrast to our previous works $[25,26]$. As discussed in detail in Refs. [27,28], one has to pay special attention to the effects of short-range correlations in a relativistic treatment of two-body matrix elements. Secondly, our calculations are performed in a full one-boson-exchange model that includes not only the long-ranged pion but also contributions from the other pseudoscalar mesons, the $\eta$ and $K$, as well as the vector mesons $\rho, \omega$, and $K^{*}$. Since nuclear structure uncertainties have been eliminated or are minimal, we can use our framework to draw conclusions regarding the sensitivity to the underlying weak baryon-baryon-meson couplings. The many-body matrix elements and two-body amplitudes are evaluated in Secs. II and III, respectively. The form of the meson exchange potential is presented in Sec. IV for the pseudoscalar and vector mesons. Section V discusses our calculation of the coupling constants. Our results are given in Sec. VI, where we discuss the influence of each meson on the total and partial rates and the proton asymmetry. Our conclusions are presented in Sec. VII.

\section{DECAY RATE AND ASYMMETRY}

The nonmesonic $\Lambda N \rightarrow N N$ decay rate is given by [26]

$$
\begin{aligned}
\Gamma_{\mathrm{nm}}= & \int \frac{d^{3} P}{(2 \pi)^{3}} \int \frac{d^{3} k}{(2 \pi)^{3}} \\
& \times \bar{\sum}(2 \pi) \delta\left(M_{H}-E_{R}-E_{1}-E_{2}\right)|\mathcal{M}|^{2},
\end{aligned}
$$

where $\mathcal{M}=\left\langle\psi_{R} ; \mathbf{P k} S M_{S} T M_{T}\left|\hat{O}_{\Lambda N \rightarrow N N}\right|_{\Lambda} A\right\rangle$ is the amplitude for the transition from an initial hypernuclear state to a final state which is divided into a two-nucleon state and a residual $(A-2)$-particle state. The quantities $M_{H}, E_{R}, E_{1}$, and $E_{2}$ are the mass of the hypernucleus, the energy of the residual $(A-2)$-particle system, and the total asymptotic energies of the emitted nucleons, respectively. A transformation to the center of mass $(\mathbf{P})$ and relative momentum $(\mathbf{k})$ of the two outgoing nucleons is already implied in Eq. (1). The sum $\bar{\Sigma}$ indicates an average over the initial hypernucleus spin projections $M_{J}$ and sum over all quantum numbers of the residual $(A-2)$-particle system, as well as the spin and isospin projections of the exiting nucleons. We follow Ref. [26] by assuming a weak-coupling scheme where the isoscalar $\Lambda$ in an orbit $\alpha_{\Lambda}=\left\{n_{\Lambda}, l_{\Lambda}, s_{\Lambda}, j_{\Lambda}, m_{\Lambda}\right\}$ couples to only the ground-state wave function of the nuclear $(A-1)$ core:

$$
\begin{aligned}
\left|{ }_{\Lambda} A\right\rangle_{T_{I} T_{3_{I}} M_{I}}^{J_{T_{I}}} & \left|\alpha_{\Lambda}\right\rangle|A-1\rangle \\
& =\sum_{m_{\Lambda} M_{C}}\left\langle j_{\Lambda} m_{\Lambda} J_{C} M_{C} \mid J_{I} M_{I}\right\rangle\left|\left(n_{\Lambda} l_{\Lambda} s_{\Lambda}\right) j_{\Lambda} m_{\Lambda}\right\rangle \\
& \times\left|J_{C} M_{C} T_{I} T_{3_{I}}\right\rangle .
\end{aligned}
$$

Employing the technique of coefficients of fractional parentage, the core wave function is further decomposed into a set of states where the nucleon in an orbit $\alpha_{N}=\left\{n_{N}, l_{N}, s_{N}, j_{N}, m_{N}\right\} \quad$ is coupled to a residual $(A-2)$-particle state:

$$
\begin{aligned}
\left|J_{C} M_{C} T_{I} T_{3_{I}}\right\rangle= & \sum_{J_{R} T_{R} j_{N}}\left\langleJ _ { C } T _ { I } \left\{\left|J_{R} T_{R}, j_{N} t_{N}\right\rangle\right.\right. \\
& \times\left[\left|J_{R}, T_{R}\right\rangle \times\left|\left(n_{N} l_{N} s_{N}\right) j_{N}, t_{N}\right\rangle\right]_{T_{I} T_{3_{I}} M_{C}} \\
= & \sum_{J_{R} T_{R} j_{N}}\left\langleJ _ { C } T _ { I } \left\{\left|J_{R} T_{R}, j_{N} t_{N}\right\rangle\right.\right. \\
& \times \sum_{M_{R} m_{N}} \sum_{T_{3_{R}} t_{3_{i}}}\left\langle J_{R} M_{R} j_{N} m_{N} \mid J_{C} M_{C}\right\rangle \\
& \times\left\langle T_{R} T_{3_{R}} t_{N} t_{3_{i}} \mid T_{I} T_{3_{I}}\right\rangle\left|J_{R} M_{R}\right\rangle\left|T_{R} T_{3_{R}}\right\rangle \\
& \times\left|\left(n_{N} l_{N} s_{N}\right) j_{N} m_{N}\right\rangle\left|t_{N} t_{3_{i}}\right\rangle
\end{aligned}
$$

where $\quad t_{N}=1 / 2$. The spectroscopic factors $S=(A-1)\left\langle J_{C} T_{I}\left\{\left|J_{R} T_{R}, j_{N} t_{N}\right\rangle^{2}\right.\right.$ appropriate for the decay of ${ }_{\Lambda}^{12} \mathrm{C}$, are taken from Ref. [29] and are listed in Table 4 of Ref. [26]. Taking into account that the $\Lambda$ decays from a $l_{\Lambda}=0$ state and working in a coupled two-body spin and isospin basis, the nonmesonic decay rate in Eq. (1) can be written as

$$
\Gamma_{\mathrm{nm}}=\Gamma_{n}+\Gamma_{p}
$$

where 


$$
\begin{aligned}
& \Gamma_{i}=\int \frac{d^{3} P}{(2 \pi)^{3}} \int \frac{d^{3} k}{(2 \pi)^{3}}(2 \pi) \delta\left(M_{H}-E_{R}-E_{1}-E_{2}\right) \sum_{S M_{S}} \sum_{J_{R} M_{R}} \sum_{T_{R} T_{3_{R}}} \frac{1}{2 J_{I}+1} \sum_{M_{I}}\left|\left\langle T_{R} T_{3_{R}} \frac{1}{2} t_{3_{i}} \mid T_{I} T_{3_{I}}\right\rangle\right|^{2} \\
& \times \sum_{T T_{3}}\left\langle T T_{3} \mid \frac{1}{2} t_{1} \frac{1}{2} t_{2}\right\rangle \sum_{m_{\Lambda} M_{C}}\left\langle j_{\Lambda} m_{\Lambda} J_{C} M_{C} \mid J_{I} M_{I}\right\rangle \sum_{j_{N}} \sqrt{A-1}\left\langleJ _ { C } T _ { I } \left\{\left|J_{R} T_{R}, j_{N} t_{N}\right\rangle \sum_{M_{R} m_{N}}\left\langle J_{R} M_{R} j_{N} m_{N} \mid J_{C} M_{C}\right\rangle\right.\right. \\
& \times \sum_{m_{l_{N}} m_{s_{N}}}\left\langle j_{N} m_{N} \mid l_{N} m_{l_{N}} \frac{1}{2} m_{s_{N}}\right\rangle \sum_{S_{0} M_{S_{0}}}\left\langle S_{0} M_{S_{0}} \mid \frac{1}{2} m_{\Lambda} \frac{1}{2} m_{s_{N}}\right\rangle \sum_{T_{0} T_{3_{0}}}\left\langle T_{0} T_{3_{0}} \mid \frac{1}{2}-\frac{1}{2} \frac{1}{2} t_{3_{i}}\right\rangle \\
& \times\left. t_{\Lambda N \rightarrow N N}\left(S, M_{S}, T, M_{T}, S_{0}, M_{S_{0}}, T_{0}, T_{3_{0}}, \mathbf{P}, \mathbf{k}\right)\right|^{2},
\end{aligned}
$$

with $t_{3_{i}}=1 / 2, t_{1}=-1 / 2, t_{2}=1 / 2$ for the $p$-induced rate $(\Lambda p \rightarrow n p)$ and $t_{3_{i}}=-1 / 2, t_{1}=-1 / 2, t_{2}=-1 / 2$ for the $n$-induced rate $(\Lambda n \rightarrow n n)$. Equation (5) is written in terms of the elementary amplitude $t_{\Lambda N \rightarrow N N}$, which accounts for the transition from an initial $\Lambda N$ state with spin (isospin) $S_{0}$ $\left(T_{0}\right)$ to a final antisymmetric $N N$ state with spin (isospin) $S(T)$. The details on how this two-body amplitude is calculated are given in the next section. Note that the $\Lambda$ has been assumed to act as a $|1 / 2-1 / 2\rangle$ isospin state which is coupled to the nucleon to total isospin $T_{0}$. As explained in the next section, this is the way to incorporate the change in isospin $\Delta I=1 / 2$ induced by the weak transition operator.

At the kinematic conditions of the $\left(\pi^{+}, K^{+}\right)$reaction carried out at KEK, the hypernucleus is created with a substantial amount of polarization in the ground state. Due to the interference between the parity-conserving and parityviolating amplitudes, the distribution of the emitted protons in the weak decay displays an angular asymmetry with respect to the polarization axis given by

$$
\sigma(\chi)=\sigma_{0}\left[1+P_{y} A_{p}(\chi)\right]
$$

where $P_{y}$ is the hypernuclear polarization, created in the production reaction, such as $\left(\pi^{+}, K^{+}\right)$at KEK and BNL or $\left(\gamma, K^{+}\right)$at CEBAF [30], and the expression for the asymmetry

$$
\begin{aligned}
A_{p}(\chi) & =\frac{3}{J+1} \frac{\operatorname{Tr}\left(\mathcal{M} \hat{S}_{y} \mathcal{M}^{\dagger}\right)}{\operatorname{Tr}\left(\mathcal{M} \mathcal{M}^{\dagger}\right)}=\frac{3}{J+1} \frac{\sum_{M_{i}} \sigma\left(M_{i}\right) M_{i}}{\sum_{M_{i}} \sigma\left(M_{i}\right)} \cos \chi \\
& =A_{p} \cos \chi
\end{aligned}
$$

shown, for instance, in Ref. [26], defines the asymmetry parameter $A_{p}$ characteristic of the hypernuclear weak decay process. The asymmetry in the distribution of protons is thus determined by the product $P_{y} A_{p}$. In the weak-coupling scheme, simple angular momentum algebra relations relate the hypernuclear polarization to the $\Lambda$ polarization:

$$
p_{\Lambda}=\left\{\begin{array}{lll}
-\frac{J}{J+1} P_{y} & \text { if } & J=J_{C}-\frac{1}{2}, \\
P_{y} & \text { if } & J=J_{C}+\frac{1}{2},
\end{array}\right.
$$

where $J_{C}$ is the spin of the nuclear core. It is convenient to introduce the intrinsic $\Lambda$ asymmetry parameter

$$
a_{\Lambda}=\left\{\begin{array}{lll}
-\frac{J+1}{J} A_{p} & \text { if } & J=J_{C}-\frac{1}{2}, \\
A_{p} & \text { if } & J=J_{C}+\frac{1}{2},
\end{array}\right.
$$

such that $P_{y} A_{p}=p_{\Lambda} a_{\Lambda}$, which is then characteristic of the elementary $\Lambda$ decay process, $\vec{\Lambda} N \rightarrow N N$, taking place in the nuclear medium.

\section{TWO-BODY AMPLITUDES}

In this section we describe how we evaluate the elementary two-body transition amplitude $t_{\Lambda N \rightarrow N N}$ which, as shown in Eq. (5), contains the dynamics of the weak decay process. In the first place, it is necessary to rewrite the product of two single particle wave functions $\left\langle\mathbf{r}_{1} \mid \alpha_{\Lambda}\right\rangle$ and $\left\langle\mathbf{r}_{2} \mid \alpha_{N}\right\rangle$ in terms of relative and center-of-mass coordinates $\mathbf{r}$ and $\mathbf{R}$. In the present work, the single particle $\Lambda$ and $\mathrm{N}$ orbits are taken to be solutions of harmonic oscillator mean field potentials with parameters $b_{\Lambda}=1.87 \mathrm{fm}$ and $b_{N}=1.64 \mathrm{fm}$, respectively, that have been adjusted to experimental separation energies and the ${ }^{12} \mathrm{C}$ charge form factor. Assuming an average size parameter $b=\left(b_{\Lambda}+b_{N}\right) / 2$, using Moshinsky brackets and working in the $L S$ representation, the product of the two harmonic oscillator single particle states $\Phi_{n l m}^{\Lambda}\left(\mathbf{r}_{1}\right)$ and $\Phi_{n^{\prime} l^{\prime} m^{\prime}}^{N}\left(\mathbf{r}_{2}\right)$ can be transformed to a linear combination of products of relative and center-of-mass wave functions. Since the $\Lambda$ is in a $l_{\Lambda}=0$ shell, we obtain

$$
\Phi_{100}^{\Lambda}\left(\frac{\mathbf{r}_{1}}{b}\right) \Phi_{100}^{N}\left(\frac{\mathbf{r}_{2}}{b}\right)=\Phi_{100}^{\text {rel }}\left(\frac{\mathbf{r}}{\sqrt{2} b}\right) \Phi_{100}^{\text {c.m. }}\left(\frac{\mathbf{R}}{b / \sqrt{2}}\right),
$$

when the nucleon is in the $s$ shell and

$$
\begin{aligned}
\Phi_{100}^{\Lambda}\left(\frac{\mathbf{r}_{1}}{b}\right) \Phi_{11 m}^{N}\left(\frac{\mathbf{r}_{2}}{b}\right)= & \frac{1}{\sqrt{2}}\left\{\Phi_{100}^{\text {rel }}\left(\frac{\mathbf{r}}{\sqrt{2} b}\right) \Phi_{11 m}^{\text {c.m. }}\left(\frac{\mathbf{R}}{b / \sqrt{2}}\right)\right. \\
& \left.-\Phi_{11 m}^{\text {rel }}\left(\frac{\mathbf{r}}{\sqrt{2} b}\right) \Phi_{100}^{\text {c.m. }}\left(\frac{\mathbf{R}}{b / \sqrt{2}}\right)\right\}
\end{aligned}
$$

when the nucleon is in the $p$ shell. 
As for the final $N N$ state, the antisymmetric state of two independently moving nucleons with center-of-mass momentum $\mathbf{P}$ and relative momentum $\mathbf{k}$ reads

$\left\langle\mathbf{R} \mathbf{r} \mid \mathbf{P} \mathbf{k} S M_{S} T M_{T}\right\rangle$

$$
=\frac{1}{\sqrt{2}} \mathrm{e}^{i \mathbf{P R}}\left[\mathrm{e}^{i \mathbf{k r}}-(-1)^{S+T} \mathrm{e}^{-i \mathbf{k r}}\right] \chi_{M_{S}}^{S} \chi_{M_{T}}^{T}
$$

To incorporate the effects of the $N N$ interaction a substitution of the plane wave by a distorted wave

$$
\mathrm{e}^{i \mathbf{k r}} \rightarrow \Psi_{\mathbf{k}}(\mathbf{r})
$$

needs to be done. Therefore, the amplitude $t_{\Lambda N \rightarrow N N}$ of Eq. (5) can be decomposed in terms of amplitudes which depend on c.m. and relative quantum numbers

$$
t_{\Lambda N \rightarrow N N}=\sum_{N_{r} L_{r} N_{R} L_{R}} X\left(N_{r} L_{r} N_{R} L_{R}, l_{\Lambda} l_{N}\right) t_{\Lambda N \rightarrow N N}^{N_{r} L_{r} N_{R} L_{R}}
$$

where $X\left(N_{r} L_{r} N_{R} L_{R}, l_{\Lambda} l_{N}\right)$ are the Moshinsky brackets which for $l_{\Lambda}=l_{N}=0$ are just $X\left(\begin{array}{llllll}1 & 0 & 1 & 0, & 0 & 0\end{array}\right)=1$, and for $l_{N}=1$ are $X\left(\begin{array}{lllll}1 & 0 & 1 & 1, & 0\end{array}\right)=1 / \sqrt{2}$ and $X\left(\begin{array}{llllll}1 & 1 & 1 & 0, & 0 & 1\end{array}\right)$ $=-1 / \sqrt{2}$, as can be seen from the decomposition of the wave function above. The matrix elements $t_{\Lambda N \rightarrow N N}^{N_{r} L_{r} N_{R} L_{R}}$ are given by

$$
\begin{aligned}
t_{\Lambda N \rightarrow N N}^{N_{r} L_{r} N_{R} L_{R}}= & \frac{1}{\sqrt{2}} \int d^{3} R \int d^{3} r \mathrm{e}^{-i \mathbf{P R}} \Psi_{\mathbf{k}}^{*}(\mathbf{r}) \chi_{M_{S}}^{\dagger S} \chi_{T_{3}}^{\dagger T} \\
& \times V(\mathbf{r}) \Phi_{N_{R} L_{R}}^{\text {c.m. }}\left(\frac{\mathbf{R}}{b / \sqrt{2}}\right) \Phi_{N_{r} L_{r}}^{\mathrm{rel}}\left(\frac{\mathbf{r}}{\sqrt{2} b}\right) \chi_{M_{S_{0}}}^{S_{0}} \chi_{T_{3_{0}}}^{T_{0}} \\
= & (2 \pi)^{3 / 2} \Phi_{N_{R} L_{R}}^{\text {c.m. }}\left(\mathbf{P} \frac{b}{\sqrt{2}}\right) t_{\mathrm{rel}}
\end{aligned}
$$

with

$$
t_{\mathrm{rel}}=\frac{1}{\sqrt{2}} \int d^{3} r \Psi_{\mathbf{k}}^{*}(\mathbf{r}) \chi_{M_{S}}^{\dagger S} \chi_{T_{3}}^{\dagger T} V(\mathbf{r}) \Phi_{N_{r} L}^{\mathrm{rel}}\left(\frac{\mathbf{r}}{\sqrt{2} b}\right) \chi_{M_{S_{0}}}^{S_{0}} \chi_{T_{3_{0}}}^{T_{0}}
$$

where, for simplicity, we have only shown the direct amplitude corresponding to the first term of Eq. (12). The function $\Phi_{N_{R} L_{R}}^{\text {c.m. }}(\mathbf{P} b / \sqrt{2})$ is the Fourier transform of the $\Lambda N$ centerof-mass wave function and $t_{\text {rel }}$ is the expectation value of the transition potential $V(\mathbf{r})$ between $\Lambda N$ and $N N$ relative wave functions. In the next section it is shown how the potential $V(\mathbf{r})$ can be decomposed as

$$
V(\mathbf{r})=\sum_{i} \sum_{\alpha} V_{\alpha}^{(i)}(r) \hat{O}_{\alpha} \hat{I}_{\alpha}^{(i)}
$$

where the index $i$ runs over the different mesons exchanged and $\alpha$ over the different spin operators, $\hat{O}_{\alpha} \in\left(\hat{1}, \boldsymbol{\sigma}_{1} \boldsymbol{\sigma}_{2}\right.$, $\left.S_{12}(\hat{\mathbf{r}})=3 \boldsymbol{\sigma}_{1} \hat{\mathbf{r}} \boldsymbol{\sigma}_{2} \hat{\mathbf{r}}-\boldsymbol{\sigma}_{1} \boldsymbol{\sigma}_{2}, \boldsymbol{\sigma}_{2} \hat{\mathbf{r}},\left[\boldsymbol{\sigma}_{1} \times \boldsymbol{\sigma}_{2}\right] \hat{\mathbf{r}}\right)$, which occur in the potential $V(\mathbf{r})$. The isospin operator $\hat{I}_{\alpha}^{(i)}$ depends on the meson and can be either $\hat{1}$ for isoscalar mesons $(\eta, \omega)$, $\boldsymbol{\tau}_{1} \boldsymbol{\tau}_{2}$ for isovector mesons $(\pi, \rho)$, or a linear combination of $\hat{1}$ and $\boldsymbol{\tau}_{1} \boldsymbol{\tau}_{2}$, with the coefficients depending on the particular spin structure piece of the potential, for isodoublet mesons $\left(K, K^{*}\right)$. The radial parts $V_{\alpha}^{(i)}(r)$ are discussed in the next section.

By performing a partial wave expansion of the final twonucleon wave function and working in the $(L S) J$-coupling scheme, the relative $\Lambda N \rightarrow N N$ amplitude $t_{\text {rel }}$ can be further decomposed:

$$
\begin{aligned}
t_{\text {rel }}= & \frac{1}{\sqrt{2}} \sum_{i} \sum_{\alpha} \sum_{L L^{\prime} J} 4 \pi i^{-L^{\prime}}\left\langle L M_{L} S M_{S} \mid J M_{J}\right\rangle Y_{L M_{L}}\left(\hat{\mathbf{k}}_{r}\right) \\
& \times\left\langle L_{r} M_{L_{r}} S_{0} M_{S_{0}} \mid J M_{J}\right\rangle\left\langle\left(L^{\prime} S\right) J M_{J}\left|\hat{O}_{\alpha}\right|\left(L_{r} S_{0}\right) J M_{J}\right\rangle \\
& \times\left\langle T T_{3}\left|\hat{I}_{\alpha}^{(i)}\right| T_{0} T_{3_{0}}\right\rangle \int r^{2} d r \Psi_{L L^{\prime}}^{* J}\left(k_{r}, r\right) V_{\alpha}^{(i)}(r) \\
& \times \Phi_{N_{r} L_{r}}^{\mathrm{rel}}\left(\frac{r}{\sqrt{2} b}\right)
\end{aligned}
$$

where $\Phi_{N_{r} L_{r}}^{\mathrm{rel}}(r / \sqrt{2} b)$ stands for the radial piece of the harmonic oscillator (H.O.) wave function. The explicit expressions for the expectation value of the spin-space piece $\left\langle\left(L^{\prime} S\right) J M_{J}\left|\hat{O}_{\alpha}\right|\left(L_{r} S_{0}\right) J M_{J}\right\rangle$ can be found in the Appendix.

The function $\Psi_{L L^{\prime}}^{J}\left(k_{r}, r\right)$ is the scattering solution of two nucleons moving under the influence of the strong interaction, for which we consider the updated version of the Reidsoft-core potential [31], given in Ref. [32], and the Nijmegen [32] $N N$ potential. Such a wave function is obtained by solving a $T$-matrix equation in momentum space and in partial wave decomposition following the method described in Ref. [33]. The tensor component of the $N N$ interaction couples relative orbital states ( $L$ and $L^{\prime}$ ) having the same parity and total angular momentum as, for instance, the ${ }^{3} S_{1}$ and ${ }^{3} D_{1}$ channels. Therefore, starting from an initial $L_{r}$ orbital momentum, the weak transition potential produces a transition to a $L^{\prime}$ value, which mixes, through the subsequent action of the strong interaction, with another value of orbital angular momentum $L$. In Table I we present all the possible final states starting from initial $\Lambda N$ states having either $L_{r}=0$ or 1 and for the central $(\Delta \mathbf{S}=0, \Delta \mathbf{L}=0)$, tensor $(\Delta \mathbf{S}=2$, $\Delta \mathbf{L}=2)$, and parity-violating $(\Delta \mathbf{S}=1, \Delta \mathbf{L}=1)$ pieces in which the transition potential can be decomposed.

In the absence of final state interactions (FSI), the $N N$ wave function in Eq. (18) would reduce to a spherical Bessel function

$$
\Psi_{L L^{\prime}}^{J}\left(k_{r}, r\right)=\delta_{L, L^{\prime}} j_{L}\left(k_{r} r\right)
$$

We note that the procedure followed here to include FSI between the two emitted nucleons differs from our previous works [34,35], where the noninteracting $N N$ pair, represented by a Bessel function in the final state, was multiplied by an average $N N$ correlation function

$$
f_{\mathrm{FSI}}(r)=1-j_{0}\left(q_{c} r\right)
$$


TABLE I. Possible ${ }^{2 S+1} L_{J}$ channels obtained in the weak decay of $p$-shell hypernuclei.

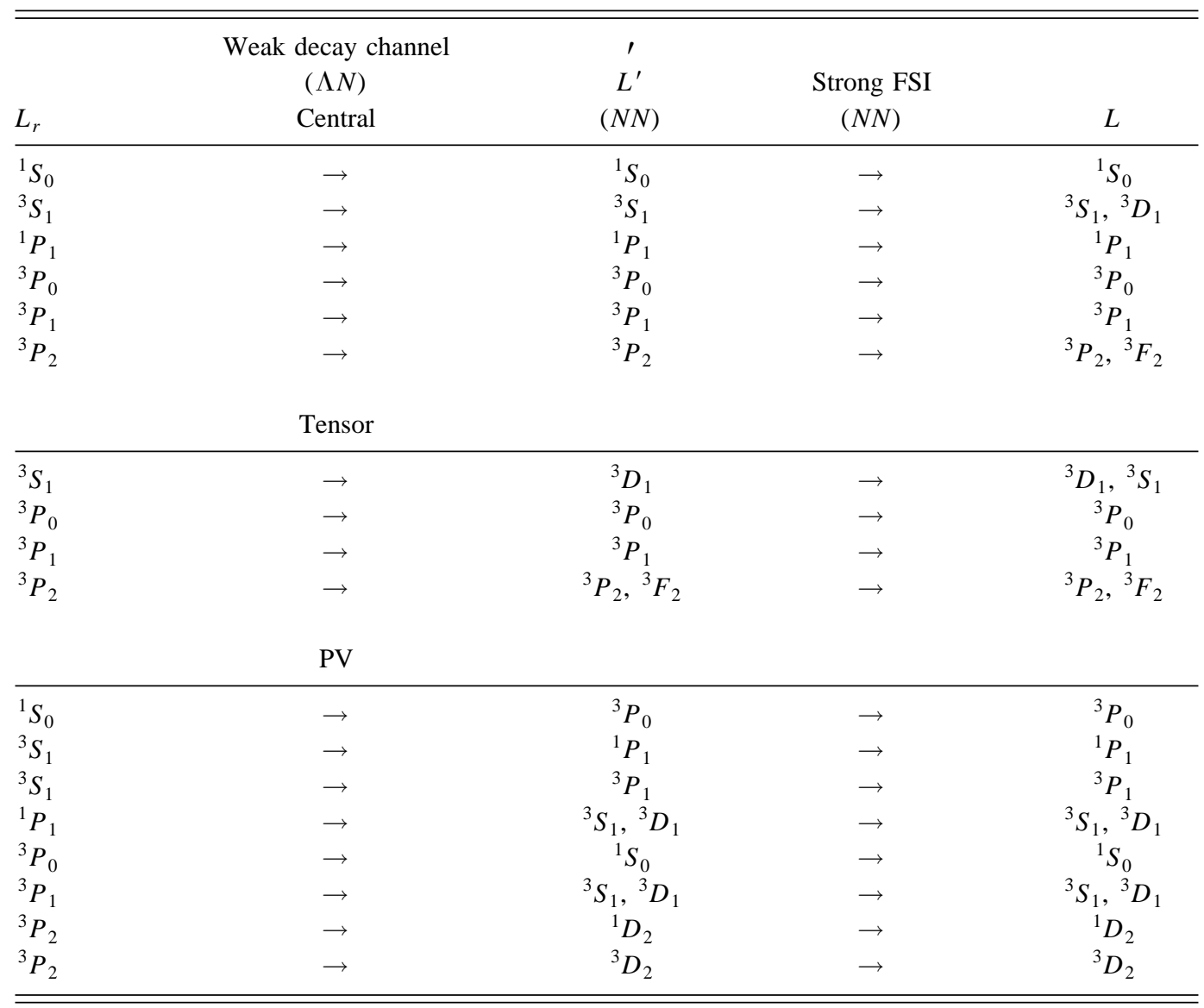

with $q_{c}=3.93 \mathrm{fm}^{-1}$, which provides a good description of nucleon pairs in ${ }^{4} \mathrm{He}$ [36] as calculated with the Reid-softcore interaction [31].

To account for the $\Lambda N$ correlations, which are absent in the independent particle model, one should replace the harmonic oscillator $\Lambda N$ wave function $\Phi_{N_{r} L_{r}}^{\text {rel }}(r)$ by a correlated $\Lambda N$ wave function that contains the effect of the strong $\Lambda N$ interaction. Such wave functions were obtained from a microscopic finite-nucleus $G$-matrix calculation [37] using the soft-core and hard-core Nijmegen models [38]. In Ref. [27] we showed that multiplying the uncorrelated harmonic oscillator $\Lambda N$ wave function with the spin-independent correlation function

$$
f(r)=\left(1-e^{-r^{2} / a^{2}}\right)^{n}+b r^{2} e^{-r^{2} / c^{2}},
$$

with $a=0.5, b=0.25, c=1.28, n=2$, yielded decay rates slightly larger than those obtained with the numerical Nijmegen-soft-core correlations but slightly smaller than those computed with the Nijmegen-hard-core potential. Since the deviations were at most $10 \%$ the above parametrization can be used as a good approximation to the full correlation function.

\section{THE MESON EXCHANGE POTENTIAL}

The transition $\Lambda N \rightarrow N N$ is assumed to proceed via the exchange of virtual mesons belonging to the ground-state pseudoscalar and vector meson octets. As displayed in Figs. 1(a) and 1(b), the transition amplitude involves a strong and a weak vertex, the later being denoted by a hatched circle.

\section{A. Pseudoscalar mesons}

While there exist several strong meson-exchange potentials which, through fits to $N N$ scattering data, provide information on the different strong $N N$-meson vertices, only the pion vertex is known experimentally in the weak sector. The weak Hamiltonian is parametrized in the form

$$
\mathcal{H}_{\Lambda N \pi}^{W}=i G_{F} m_{\pi}^{2} \bar{\psi}_{N}\left(A_{\pi}+B_{\pi} \gamma_{5}\right) \tau \phi^{\pi} \psi_{\Lambda}\left(\begin{array}{l}
0 \\
1
\end{array}\right)
$$

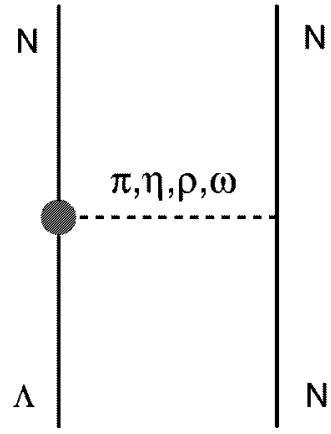

(a)

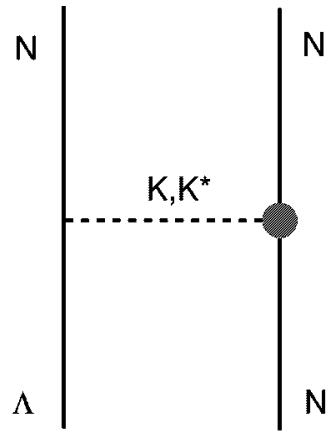

(b)
FIG. 1. Nonstrange (a) and strange (b) meson exchange contribution to the $\Lambda N \rightarrow N N$ weak transition potential. The weak vertex is indicated by the circle. 

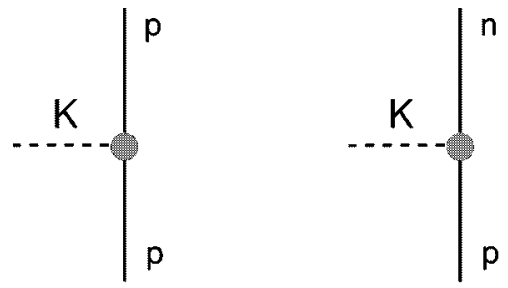

$D_{K}^{P V}+D_{K}^{P C} \gamma_{5}$

$$
C_{K}^{P V}+C_{K}^{P C} \gamma_{5}
$$

where $G_{F} m_{\pi}^{2}=2.21 \times 10^{-7}$ is the weak-coupling constant. The empirical constants $A_{\pi}=1.05$ and $B_{\pi}=-7.15$, adjusted to the observables of the free $\Lambda$ decay, determine the strength of the parity-violating and parity-conserving amplitudes, respectively. The nucleon, lambda, and pion fields are given by $\psi_{\mathrm{N}}, \psi_{\Lambda}$, and $\boldsymbol{\phi}^{\pi}$, respectively, while the isospin spurion $\left(\begin{array}{l}0 \\ 1\end{array}\right)$ is included to enforce the empirical $\Delta I=1 / 2$ rule observed in the decay of a free $\Lambda$.

For the strong vertex, we take the usual pseudoscalar coupling

$$
\mathcal{H}_{N N \pi}^{S}=i g_{N N \pi} \bar{\psi}_{N} \gamma_{5} \boldsymbol{\tau} \boldsymbol{\phi}^{\pi} \psi_{N}
$$

which is equivalent to the pseudovector coupling when free spinors are used in the evaluation of the transition amplitude. The nonrelativistic reduction of the free space Feynman amplitude is then associated with the transition potential. In momentum space, one obtains

$$
V_{\pi}(\mathbf{q})=-G_{F} m_{\pi}^{2} \frac{g}{2 M}\left(\hat{A}+\frac{\hat{B}}{2 \bar{M}} \boldsymbol{\sigma}_{1} \mathbf{q}\right) \frac{\boldsymbol{\sigma}_{2} \mathbf{q}}{\mathbf{q}^{2}+\mu^{2}}
$$

where $\mathbf{q}$ is the momentum carried by the pion directed towards the strong vertex, $g=g_{N N \pi}$ the strong-coupling constant for the $N N \pi$ vertex, $\mu$ the pion mass, $M$ the nucleon mass, and $\bar{M}$ the average between the nucleon and $\Lambda$ masses. The operators $\hat{A}$ and $\hat{B}$, which contain the isospin dependence of the potential, read

$$
\begin{aligned}
& \hat{A}=A_{\pi} \boldsymbol{\tau}_{1} \boldsymbol{\tau}_{2}, \\
& \hat{B}=B_{\pi} \boldsymbol{\tau}_{1} \boldsymbol{\tau}_{2} .
\end{aligned}
$$

We note that the nonrelativistic approach of the present work differs from our previous works [25,26], which were based on a relativistic formalism. It was found that the supression of the matrix elements due to short-range correlations was larger by about a factor of 2 to what was obtained in standard nonrelativistic calculations $[14,15,17,18]$. In Ref. [27] it was shown that, if one uses the same nonrelativistic correlation function, the relativistic and nonrelativistic schemes were not giving the same correlated potential obtained through the standard nonrelativistic reduction. In the relativistic approach, the correlation function was applied to the Feynman amplitude before the nonrelativistic reduction was carried out, whereas in the nonrelativistic procedure the correlation function was applied after the reduction of the free Feynman amplitude was obtained. The difference between the two methods was studied in Ref. [28], where it was shown explicitly that the relativistic framework together with a standard nonrelativistic correlation function lead to additional contributions in the correlated transition potential which produced the larger supression of the decay rates reported in Refs. [25,26]. For lack of relativistic correlation functions we adopt a nonrelativistic formalism in this paper.

The other mesons of the pseudoscalar octet are the isosinglet eta $(\eta)$ and the isodoublet kaon $(K)$. The strong and weak vertices for these mesons are

$$
\begin{gathered}
\mathcal{H}_{N N \eta}^{S}=i g_{N N \eta} \bar{\psi}_{N} \gamma_{5} \phi^{\eta} \psi_{N} \\
\mathcal{H}_{\Lambda N \eta}^{W}=i G_{F} m_{\pi}^{2} \bar{\psi}_{N}\left(A_{\eta}+B_{\eta} \gamma_{5}\right) \phi^{\eta} \psi_{\Lambda}\left(\begin{array}{l}
0 \\
1
\end{array}\right) \\
\mathcal{H}_{\Lambda N K}^{S}=i g_{\Lambda N K} \bar{\psi}_{N} \gamma_{5} \phi^{K} \psi_{\Lambda}, \\
\mathcal{H}_{N N K}^{W}=i G_{F} m_{\pi}^{2}\left[\bar{\psi}_{N}\left(\begin{array}{l}
0 \\
1
\end{array}\right)\left(C_{K}^{\mathrm{PV}}+C_{K}^{\mathrm{PC}} \gamma_{5}\right)\left(\phi^{K}\right)^{\dagger} \psi_{N}+\bar{\psi}_{N} \psi_{N}\right. \\
\left.\times\left(D_{K}^{\mathrm{PV}}+D_{K}^{\mathrm{PC}} \gamma_{5}\right)\left(\phi^{K}\right)^{\dagger}\left(\begin{array}{l}
0 \\
1
\end{array}\right)\right]
\end{gathered}
$$

where the weak-coupling constants cannot be derived from experiment. In the present work we adopt the approach of Refs. [18,42], presented in the next section.

We note that the isospurion $\left(\begin{array}{l}0 \\ 1\end{array}\right)$ appearing in the former equations is used to enforce the empirical $\Delta I=1 / 2$ rule. The particular structure of the $K$ weak couplings reproduce the vertices shown in Fig. 2.

The corresponding nonrelativistic potentials for the transition $\Lambda N \rightarrow N N$ are analogous to Eq. (24) but making the following replacements:

$$
\begin{gathered}
g \rightarrow g_{N N \eta}, \\
\mu \rightarrow m_{\eta}, \\
\hat{A} \rightarrow A_{\eta}, \\
\hat{B} \rightarrow B_{\eta},
\end{gathered}
$$

in the case of $\eta$ exchange, and

$$
\begin{gathered}
g \rightarrow g_{\Lambda N K}, \\
\mu \rightarrow m_{K}, \\
\hat{A} \rightarrow\left(\frac{C_{K}^{\mathrm{PV}}}{2}+D_{K}^{\mathrm{PV}}+\frac{C_{K}^{\mathrm{PV}}}{2} \tau_{1} \tau_{2}\right) \frac{M}{\bar{M}}, \\
\hat{B} \rightarrow\left(\frac{C_{K}^{\mathrm{PC}}}{2}+D_{K}^{\mathrm{PC}}+\frac{C_{K}^{\mathrm{PC}}}{2} \tau_{1} \boldsymbol{\tau}_{2}\right),
\end{gathered}
$$


in the case of $K$ exchange, where the factor $M / \bar{M}$ corrects for the fact that the nonrelativistic reduction of the strong $\Lambda N K$ vertex gives a factor $1 / \bar{M}$ instead of $1 / M$. Performing a Fourier transform of the general expression given in Eq. (24) and introducing the tensor operator $S_{12}(\hat{\mathbf{r}})=3 \boldsymbol{\sigma}_{1} \hat{\mathbf{r}} \boldsymbol{\sigma}_{2} \hat{\mathbf{r}}-\boldsymbol{\sigma}_{1} \boldsymbol{\sigma}_{2}$, it is easy to obtain the corresponding transition potential in coordinate space, which can be divided into central, tensor, and parity-violating pieces. The explicit expressions are given at the end of this section.

\section{B. Vector mesons}

A number of theoretical studies in recent years have investigated the contribution of the $\rho$-meson to the $\Lambda N \rightarrow N N$ process $[15,16,39]$. The weak $\Lambda N \rho$ and strong $N N \rho$ vertices are given by [15]

$$
\begin{gathered}
\mathcal{H}_{\Lambda N \rho}^{W}=G_{F} m_{\pi}^{2} \bar{\psi}_{N}\left(\alpha_{\rho} \gamma^{\mu}-\beta_{\rho} i \frac{\sigma^{\mu \nu} q_{\nu}}{2 \bar{M}}+\varepsilon_{\rho} \gamma^{\mu} \gamma_{5}\right) \tau \rho_{\mu} \psi_{\Lambda}\left(\begin{array}{l}
0 \\
1
\end{array}\right), \\
\mathcal{H}_{N N \rho}^{S}=\bar{\psi}_{N}\left(g_{N N \rho}^{V} \gamma^{\mu}+i \frac{g_{N N \rho}^{T}}{2 M} \sigma^{\mu \nu} q_{\nu}\right) \tau \rho_{\mu} \psi_{N}
\end{gathered}
$$

respectively, where the four momentum transfer $q$ is directed towards the strong vertex. The values of the strong- and weak-coupling constants are given in the next section.

The nonrelativistic reduction of the Feynman amplitude gives the following $\rho$-meson transition potential:

$$
\begin{aligned}
V_{\rho}(\mathbf{q})= & G_{F} m_{\pi}^{2}\left(F_{1} \hat{\alpha}-\frac{(\hat{\alpha}+\hat{\beta})\left(F_{1}+F_{2}\right)}{4 M \bar{M}}\left(\boldsymbol{\sigma}_{1} \times \mathbf{q}\right)\left(\boldsymbol{\sigma}_{2} \times \mathbf{q}\right)\right. \\
& \left.+i \frac{\hat{\varepsilon}\left(F_{1}+F_{2}\right)}{2 M}\left(\boldsymbol{\sigma}_{1} \times \boldsymbol{\sigma}_{2}\right) \mathbf{q}\right) \frac{1}{\mathbf{q}^{2}+\mu^{2}}
\end{aligned}
$$

with $\mu=m_{\rho}, F_{1}=g_{N N \rho}^{V}, F_{2}=g_{N N \rho}^{T}$, and the operators $\hat{\alpha}$, $\hat{\beta}$, and $\hat{\varepsilon}$ :

$$
\begin{gathered}
\hat{\alpha}=\alpha_{\rho} \tau_{1} \tau_{2}, \\
\hat{\beta}=\beta_{\rho} \tau_{1} \tau_{2}, \\
\hat{\varepsilon}=\varepsilon_{\rho} \tau_{1} \tau_{2}
\end{gathered}
$$

contain the isospin structure. Using the relation $\left(\boldsymbol{\sigma}_{1}\right.$ $\times \mathbf{q})\left(\boldsymbol{\sigma}_{2} \times \mathbf{q}\right)=\left(\boldsymbol{\sigma}_{1} \boldsymbol{\sigma}_{2}\right) \mathbf{q}^{2}-\left(\boldsymbol{\sigma}_{1} \mathbf{q}\right)\left(\boldsymbol{\sigma}_{2} \mathbf{q}\right)$ and performing a Fourier transform of $V_{\rho}(\mathbf{q})$, one obtains the corresponding transition potential in coordinate space, which, as in $\pi$ exchange, can be divided into central, tensor and parityviolating pieces. Furthermore, the $\rho$-meson central potential can be further decomposed into a spin-independent and a spin-dependent part [34]. Due to the different models employed for the weak $\Lambda N \rho$ vertex [15,16,39], different calculations have yielded widely varying results. However, all studies until now have only included the tensor piece of the parity-conserving $\rho$-exchange term motivated, in part, by the observation that this is the most important contribution to the $\pi$-exchange potential. We recently demonstrated [34] that the central piece of the $\rho$ exchange is in fact larger than its tensor interaction, an observation that can be traced to the fact that the $\rho$-exchange diagram has a much shorter range than the $\pi$-exchange potential. It is therefore important to explicitly keep all pieces of the potential for the vector mesons.

The other vector mesons considered in this work are the isoscalar $\omega$ and the isodoublet $K^{*}$, for which the weak and strong vertices can be written as

$$
\begin{aligned}
& \mathcal{H}_{N N \omega}^{S}= \bar{\psi}_{N}\left(g_{N N \omega}^{V} \gamma^{\mu}+i \frac{g_{N N \omega}^{T}}{2 M} \sigma^{\mu \nu} q_{\nu}\right) \phi_{\mu}^{\omega} \psi_{N} \\
& \mathcal{H}_{\Lambda N \omega}^{W}= G_{F} m_{\pi}^{2} \bar{\psi}_{N}\left(\alpha_{\omega} \gamma^{\mu}-\beta_{\omega} i \frac{\sigma^{\mu \nu} q_{\nu}}{2 \bar{M}}+\varepsilon_{\omega} \gamma^{\mu} \gamma_{5}\right) \phi_{\mu}^{\omega} \psi_{\Lambda}\left(\begin{array}{l}
0 \\
1
\end{array}\right) \\
& \mathcal{H}_{\Lambda N K^{*}}^{S}=\bar{\psi}_{N}\left(g_{\Lambda N K^{*}}^{V} \gamma^{\mu}+i \frac{g_{\Lambda N K^{*}}^{T}}{2 \bar{M}} \sigma^{\mu \nu} q_{\nu}\right) \phi_{\mu}^{K^{*}} \psi_{\Lambda} \\
& \mathcal{H}_{N N K^{*}}^{W}= G_{F} m_{\pi}^{2}\left(\left[C_{K^{*}}^{P C, V} \bar{\psi}_{N}\left(\begin{array}{l}
0 \\
1
\end{array}\right)\left(\phi_{\mu}^{K^{*}}\right)^{\dagger} \gamma^{\mu} \psi_{N}\right.\right. \\
&\left.+D_{K^{*}}^{\mathrm{PC}, V} \bar{\psi}_{N} \gamma^{\mu} \psi_{N}\left(\phi_{\mu}^{K^{*}}\right)^{\dagger}\left(\begin{array}{l}
0 \\
1
\end{array}\right)\right]+\left[C_{K^{*}}^{\mathrm{PC}, T} \bar{\psi}_{N}\left(\begin{array}{l}
0 \\
1
\end{array}\right)\right. \\
& \times\left(\phi_{\mu}^{K^{*}}\right)^{\dagger}(-i) \frac{\sigma^{\mu \nu} q_{\nu}}{2 M} \psi_{N}+D_{K^{*}}^{\mathrm{PC}, T} \bar{\psi}_{N} \\
&\left.\times(-i) \frac{\sigma^{\mu \nu} q_{\nu}}{2 M} \psi_{N}\left(\phi_{\mu}^{K^{*}}\right)^{\dagger}\left(\begin{array}{l}
0 \\
1
\end{array}\right)\right] \\
&+\left[C_{K^{*}}^{\mathrm{PV}} \bar{\psi}_{N}\left(\begin{array}{l}
0 \\
1
\end{array}\right)\left(\phi_{\mu}^{K^{*}}\right)^{\dagger} \gamma^{\mu} \gamma_{5} \psi_{N}\right. \\
&\left.\left.+D_{K^{*}}^{\mathrm{PV}} \bar{\psi}_{N} \gamma^{\mu} \gamma_{5} \psi_{N}\left(\phi_{\mu}^{K^{*}}\right)^{\dagger}\left(\begin{array}{l}
0 \\
1
\end{array}\right)\right]\right)
\end{aligned}
$$

Note that the $K^{*}$ weak vertex has the same structure as the $K$ vertex, the only difference being the parity conserving contribution which has two terms, related to the vector and tensor couplings. The nonrelativistic potential can be obtained from the general expression given in Eq. (34) making the following replacements:

$$
\begin{gathered}
\mu \rightarrow m_{\omega}, \\
F_{1} \rightarrow g_{N N \omega}^{V}, \\
F_{2} \rightarrow g_{N N \omega}^{T}, \\
\hat{\alpha} \rightarrow \alpha_{\omega}, \\
\hat{\beta} \rightarrow \beta_{\omega}, \\
\hat{\varepsilon} \rightarrow \varepsilon_{\omega},
\end{gathered}
$$

in the case of $\omega$ exchange, and

$$
\mu \rightarrow m_{K^{*}},
$$




$$
\begin{gathered}
F_{1} \rightarrow g_{\Lambda N K^{*}}^{V}, \\
F_{2} \rightarrow g_{\Lambda N K^{*}}^{T}, \\
\hat{\alpha} \rightarrow \frac{C_{K^{*}}^{\mathrm{PC}, V}}{2}+D_{K^{*}}^{\mathrm{PC}, V}+\frac{C_{K^{*}}^{\mathrm{PC}, V}}{2} \tau_{1} \boldsymbol{\tau}_{2}, \\
\hat{\beta} \rightarrow \frac{C_{K^{*}}^{\mathrm{PC}, T}}{2}+D_{K^{*}}^{\mathrm{PC}, T}+\frac{C_{K^{*}}^{\mathrm{PC}, T}}{2} \boldsymbol{\tau}_{1} \boldsymbol{\tau}_{2}, \\
\hat{\varepsilon} \rightarrow\left(\frac{C_{K^{*}}^{\mathrm{PV}}}{2}+D_{K^{*}}^{\mathrm{PV}}+\frac{C_{K^{*}}^{\mathrm{PV}}}{2} \boldsymbol{\tau}_{1} \boldsymbol{\tau}_{2}\right) \frac{M}{\bar{M}},
\end{gathered}
$$

for the $K^{*}$ meson exchange.

\section{General form of the potential}

The Fourier transform of the general Eqs. (24) and (34) leads to a potential in configuration space which can be cast into the form

$$
\begin{aligned}
V(\mathbf{r})= & \sum_{i} \sum_{\alpha} V_{\alpha}^{(i)}(\mathbf{r})=\sum_{i} \sum_{\alpha} V_{\alpha}^{(i)}(r) \hat{O}_{\alpha} \hat{I}_{\alpha}^{(i)} \\
= & \sum_{i}\left\{V_{C}^{(i)}(r) \hat{I}_{C}^{(i)}+V_{S S}^{(i)}(r) \boldsymbol{\sigma}_{1} \boldsymbol{\sigma}_{2} \hat{I}_{S S}^{(i)}+V_{T}^{(i)}(r) S_{12}(\hat{\mathbf{r}}) \hat{I}_{T}^{(i)}\right. \\
& \left.+\left[n^{i} \boldsymbol{\sigma}_{2} \cdot \hat{\mathbf{r}}+\left(1-n^{i}\right)\left[\boldsymbol{\sigma}_{1} \times \boldsymbol{\sigma}_{2}\right] \cdot \hat{\mathbf{r}}\right] V_{\mathrm{PV}}^{(i)}(r) \hat{I}_{\mathrm{PV}}^{(i)}\right\},
\end{aligned}
$$

where the index $i$ runs over the different mesons exchanged ( $i=1, \ldots, 6$ represents $\left.\pi, \eta, K, \rho, \omega, K^{*}\right)$ and $\alpha$ over the different spin operators denoted by $C$ (central spin independent), $S S$ (central spin dependent), $T$ (tensor), and $P V$ (parity violating). In the above expression, particle 1 refers to the $\Lambda$ and $n^{i}=1$ (0) for pseudoscalar (vector) mesons. In the case of isovector mesons $(\pi, \rho)$ the isospin factor is $\boldsymbol{\tau}_{1} \boldsymbol{\tau}_{2}$ and for isoscalar mesons $(\eta, \omega)$ this factor is just $\hat{1}$ for all spin structure pieces of the potential. In the case of isodoublet mesons $\left(K, K^{*}\right)$ there are contributions proportional to $\hat{1}$ and to $\boldsymbol{\tau}_{1} \boldsymbol{\tau}_{2}$ that depend on the coupling constants and, therefore, on the spin structure piece of the potential denoted by $\alpha$. For $K$ exchange we have

$$
\begin{gathered}
\hat{I}_{C}^{(3)}=0, \\
\hat{I}_{S S}^{(3)}=\hat{I}_{T}^{(3)}=\frac{C_{K}^{\mathrm{PC}}}{2}+D_{K}^{\mathrm{PC}}+\frac{C_{K}^{\mathrm{PC}}}{2} \tau_{1} \boldsymbol{\tau}_{2}, \\
\hat{I}_{\mathrm{PV}}^{(3)}=\frac{C_{K}^{\mathrm{PV}}}{2}+D_{K}^{\mathrm{PV}}+\frac{C_{K}^{\mathrm{PV}}}{2} \boldsymbol{\tau}_{1} \boldsymbol{\tau}_{2},
\end{gathered}
$$

and for $K^{*}$ exchange,

$$
\begin{aligned}
\hat{I}_{C}^{(6)}= & \frac{C_{K^{*}}^{\mathrm{PC}, V}}{2}+D_{K^{*}}^{\mathrm{PC}, V}+\frac{C_{K^{*}}^{\mathrm{PC}, V}}{2} \boldsymbol{\tau}_{1} \boldsymbol{\tau}_{2}, \\
\hat{I}_{S S}^{(6)}=\hat{I}_{T}^{(6)}= & \frac{\left(C_{K^{*}}^{\mathrm{PC}, V}+C_{K^{*}}^{\mathrm{PC}, T}\right)}{2}+\left(D_{K^{*}}^{\mathrm{PC}, V}+D_{K^{*}}^{\mathrm{PC}, T}\right) \\
& +\frac{\left(C_{K^{*}}^{\mathrm{PC}, V}+C_{K^{*}}^{\mathrm{PC}, T}\right)}{2} \boldsymbol{\tau}_{1} \boldsymbol{\tau}_{2}, \\
\hat{I}_{\mathrm{PV}}^{(6)}= & \frac{C_{K^{*}}^{\mathrm{PV}}}{2}+D_{K^{*}}^{\mathrm{PV}}+\frac{C_{\mathrm{K} *}^{\mathrm{PV}}}{2} \boldsymbol{\tau}_{1} \boldsymbol{\tau}_{2} .
\end{aligned}
$$

The different pieces $V_{\alpha}^{(i)}$, with $\alpha=C, S S, T, \mathrm{PV}$, are given by

$$
\begin{gathered}
V_{C}^{(i)}(r)=K_{C}^{(i)} \frac{e^{-\mu_{i} r}}{4 \pi r} \equiv K_{C}^{(i)} V_{C}\left(r, \mu_{i}\right) \\
V_{S S}^{(i)}(r)=K_{S S}^{(i)} \frac{1}{3}\left[\mu_{i}^{2} \frac{e^{-\mu_{i} r}}{4 \pi r}-\delta(\mathbf{r})\right] \equiv K_{S S}^{(i)} V_{S S}\left(r, \mu_{i}\right) \\
V_{T}^{(i)}(r)=K_{T}^{(i)} \frac{1}{3} \mu_{i}^{2} \frac{e^{-\mu_{i} r}}{4 \pi r}\left(1+\frac{3}{\mu_{i} r}+\frac{3}{\left(\mu_{i} r\right)^{2}}\right) \equiv K_{T}^{(i)} V_{T}\left(r, \mu_{i}\right) \\
V_{\mathrm{PV}}^{(i)}(r)=K_{\mathrm{PV}}^{(i)} \mu_{i} \frac{e^{-\mu_{i} r}}{4 \pi r}\left(1+\frac{1}{\mu_{i} r}\right) \equiv K_{\mathrm{PV}}^{(i)} V_{\mathrm{PV}}\left(r, \mu_{i}\right)
\end{gathered}
$$

where $\mu_{i}$ denotes the mass of the different mesons. It is these expressions that are inserted in Eq. (18) to compute $t_{\text {rel }}$ numerically. The expressions for $K_{\alpha}^{(i)}$, which contain factors and coupling constants, are given in Table II.

A monopole form factor $F_{i}\left(\mathbf{q}^{2}\right)=\left(\Lambda_{i}^{2}-\mu_{i}^{2}\right) /\left(\Lambda_{i}^{2}+\mathbf{q}^{2}\right)$ is used at each vertex, where the value of the cutoff $\Lambda_{i}$ depends on the meson. We take the values of the Jülich $Y N$ interaction [40], displayed in Table III of Sec. V, since the Nijmegen model distinguishes form factors only in terms of the transition channel. The use of form factors leads to the following regularization for each meson:

$$
\begin{aligned}
& V_{C}\left(r ; \mu_{i}\right) \rightarrow V_{C}\left(r ; \mu_{i}\right)-V_{C}\left(r ; \Lambda_{i}\right) \\
&-\Lambda_{i} \frac{\Lambda_{i}^{2}-\mu_{i}^{2}}{2} \frac{e^{-\Lambda_{i} r}}{4 \pi}\left(1-\frac{2}{\Lambda_{i} r}\right) \\
& V_{S S}\left(r ; \mu_{i}\right) \rightarrow V_{S S}\left(r ; \mu_{i}\right)-V_{S S}\left(r ; \Lambda_{i}\right) \\
&-\Lambda_{i} \frac{\Lambda_{i}^{2}-\mu_{i}^{2}}{2} \frac{e^{-\Lambda_{i} r}}{4 \pi}\left(1-\frac{2}{\Lambda_{i} r}\right) \\
& V_{T}\left(r ; \mu_{i}\right) \rightarrow V_{T}\left(r ; \mu_{i}\right)-V_{T}\left(r ; \Lambda_{i}\right) \\
&-\Lambda_{i} \frac{\Lambda_{i}^{2}-\mu_{i}^{2}}{2} \frac{e^{-\Lambda_{i} r}}{4 \pi}\left(1+\frac{1}{\Lambda_{i} r}\right) \\
& V_{\mathrm{PV}}\left(r ; \mu_{i}\right) \rightarrow V_{\mathrm{PV}}\left(r ; \mu_{i}\right)-V_{\mathrm{PV}}\left(r ; \Lambda_{i}\right)-\frac{\Lambda_{i}^{2}-\mu_{i}^{2}}{2} \frac{e^{-\Lambda_{i} r}}{4 \pi}
\end{aligned}
$$


TABLE II. Constants appearing in weak transition potential for the different mesons.

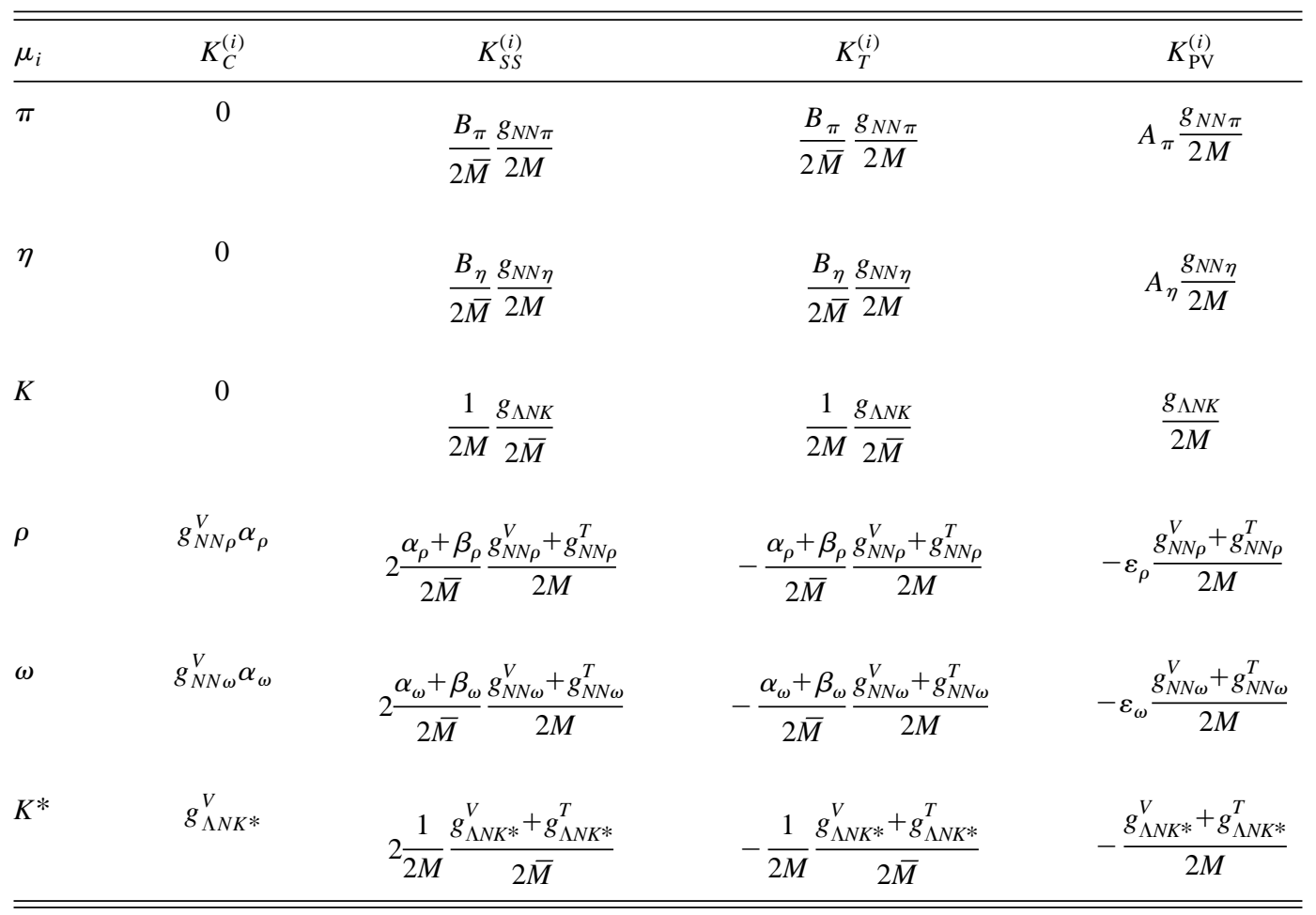

where $V_{\alpha}\left(r ; \Lambda_{i}\right)$ has the same structure as $V_{\alpha}\left(r ; \mu_{i}\right)$, defined in Eqs. (45)-(48), but replacing the meson mass $\mu_{i}$ by the corresponding cutoff mass $\Lambda_{i}$.

\section{THE WEAK-COUPLING CONSTANTS}

The starting point for describing the weak decay of strange particles has been the fundamental Cabbibo Hamiltonian based on the current $\otimes$ current assumption

$$
H=\frac{G_{F}}{\sqrt{2}} \int d^{3} x J^{\alpha}(x) J_{\alpha}^{\dagger}(x)+\text { H.c. }
$$

with

$$
\begin{aligned}
J_{\alpha}(x)= & \bar{\Psi}_{e}(x) \gamma_{\alpha}\left(1-\gamma_{5}\right) \Psi_{\nu_{e}}(x)+\bar{\Psi}_{\mu}(x) \gamma_{\alpha} \\
& \times\left(1-\gamma_{5}\right) \Psi_{\nu_{\mu}}(x)+\bar{u}(x) \gamma_{\alpha}\left(1-\gamma_{5}\right) \\
& \times\left[d(x) \cos \theta_{C}+s(x) \sin \theta_{C}\right]
\end{aligned}
$$

where $\theta_{C}$ is the Cabbibo angle, $G_{F}$ the weak-coupling constant, and we take the Bjorken and Drell convention for the definition of $\gamma_{5}$ [41]. As is well known, terms proportional to $\cos \theta_{C}$ describe, for instance, the neutron $\beta$ decay while the contributions proportional to $\sin \theta_{C}$ lead to the semileptonic decay of hyperons and kaons. The $\Delta S=1$ nonleptonic decays are governed by terms proportional to $\sin \theta_{C} \cos \theta_{C}$ which consist of products of a current between $u$ and $d$ quarks $(\Delta I=1)$ and a current betwen $u$ and $s$ quarks $(\Delta I=1 / 2)$. Thus, since terms in $\sin \theta_{C} \cos \theta_{C}$ describe transitions with $\Delta I=1 / 2$ and $3 / 2$ with equal probability, the empirical $\Delta I=$
$1 / 2$ rule indicates the presence of some dynamical effect related to QCD corrections that suppresses the $\Delta I=3 / 2$ components of the Hamiltonian.

In order to obtain hadronic weak matrix elements of the kind $\left\langle M B^{\prime}\left|H_{w}\right| B\right\rangle$, where $M$ can stand for pseudoscalar or vector mesons and $B$ for baryons, it has been convenient to express the effective weak Hamiltonian in terms of the $\mathrm{SU}(6)_{w}$ symmetry that unites them.

The $\Delta S=1$ weak nonleptonic Hamiltonian can be written in $\mathrm{SU}(3)$ tensor notation:

$$
H_{w}=\frac{G_{F}}{2 \sqrt{2}} \cos \theta_{C} \sin \theta_{C}\left\{J_{\mu 1}^{2}, J_{3}^{\mu 1}\right\}+\text { H.c. }
$$

where $J_{\mu j}^{i}=\left(V_{\mu}-A_{\mu}\right)_{j}^{i}$ is the weak hadronic current with $\mathrm{SU}(3)$ indices $i$ and $j$. As shown in Refs. $[18,42,43]$ the weak vector and axial currents can be expressed in terms of $\mathrm{SU}(6)_{w}$ currents. Since the Hamiltonian is the product of two currents, each belonging to the $\mathbf{3 5}$ representation, one can expand

$35 \otimes 35=1_{s} \oplus 35_{s} \oplus 189_{s} \oplus 405_{s} \oplus 35_{a} \oplus 280_{a} \oplus \overline{280_{a}}$,

which allows extraction of the parity-violating (PV) and parity-conserving $(\mathrm{PC})$ pieces of the Hamiltonian:

$$
\begin{gathered}
H_{\mathrm{PC}}: 1_{s} \oplus 35_{s} \oplus 189_{s} \oplus 405_{s} \\
H_{\mathrm{PV}}: 35_{a} \oplus 280_{a} \oplus \overline{280_{a}} .
\end{gathered}
$$

Each of the possible ways of coupling baryons to mesons within the $\mathrm{SU}(6)_{w}$ symmetry introduces a reduced matrix element that can either be fitted to experimental data or cal- 
TABLE III. Nijmegen [38] (Jülich [40]) strong-coupling constants, weak-coupling constants [18], and cutoff parameters for the different mesons. The weak couplings are in units of $G_{F} m_{\pi}^{2}=2.21 \times 10^{-7}$. For the kaon and the $\rho$ meson we also quote the weak couplings obtained by Ref. [46] and Ref. [16], respectively.

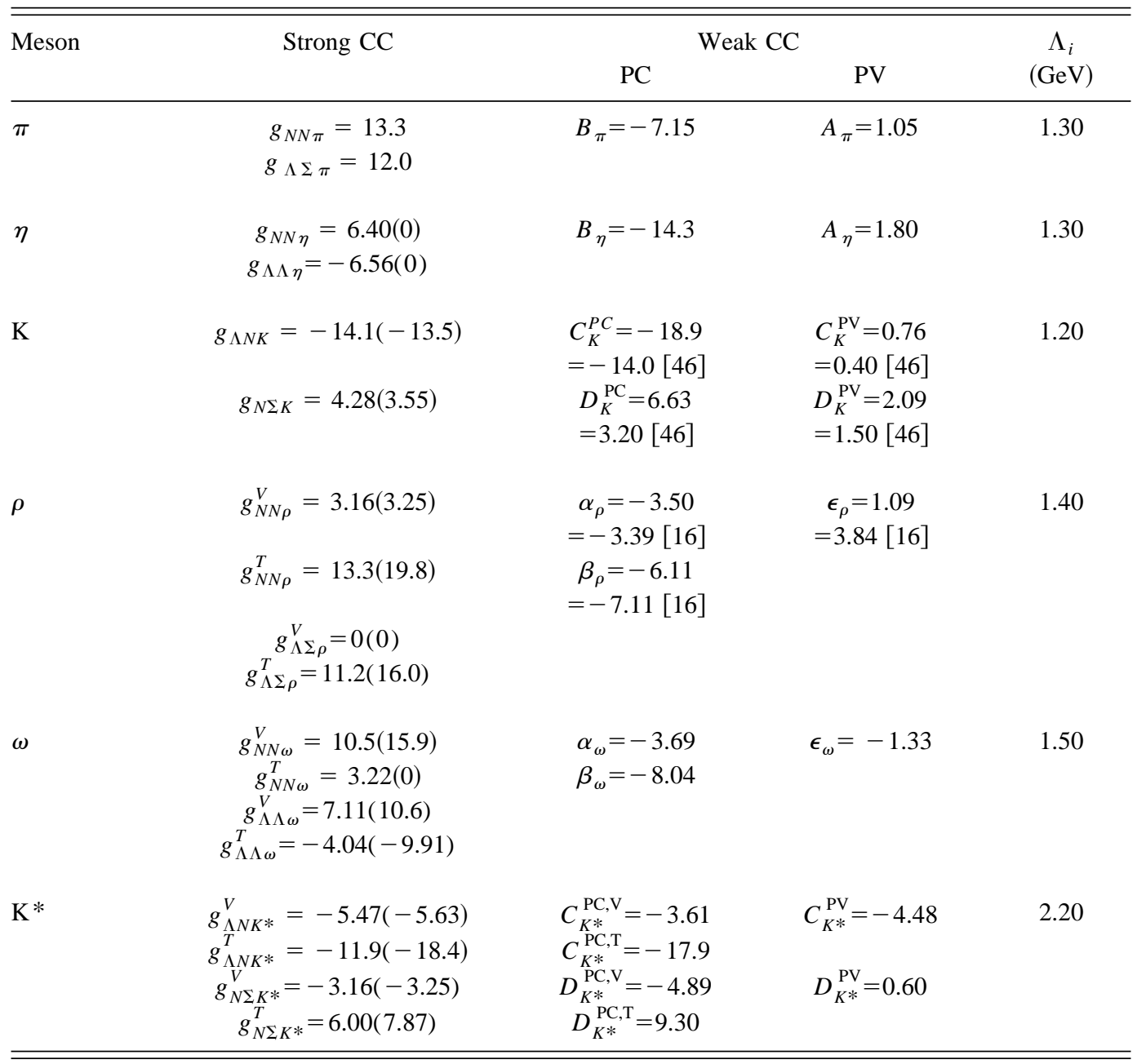

culated microscopically from quark models. Below we discuss the PV and PC amplitudes separately.

\section{A. The PV amplitudes}

The traditional approximation employed to obtain the PV amplitudes for the nonleptonic decays $B \rightarrow B^{\prime}+M$ has been the use of the soft-meson reduction theorem:

$$
\begin{aligned}
\lim _{q \rightarrow 0}\left\langle B^{\prime} M_{i}(q)\left|H_{\mathrm{PV}}\right| B\right\rangle & =-\frac{i}{F_{\pi}}\left\langle B^{\prime}\left|\left[F_{i}^{5}, H_{\mathrm{PV}}\right]\right| B\right\rangle \\
& =-\frac{i}{F_{\pi}}\left\langle B^{\prime}\left|\left[F_{i}, H_{\mathrm{PC}}\right]\right| B\right\rangle,
\end{aligned}
$$

where $q$ is the momentum of the meson and $F_{i}$ is an $\mathrm{SU}(3)$ generator whose action on a baryon $B_{j}$ gives

$$
F_{i}\left|B_{j}\right\rangle=i f_{i j k}\left|B_{k}\right\rangle \text {. }
$$

Since the weak Hamiltonian $H_{w}$ is assumed to transform like the sixth component of an octet, a term such as $\left\langle B_{k}\left|H_{w}^{6}\right| B_{j}\right\rangle$ can be expressed as

$$
\left\langle B_{k}\left|H_{w}^{6}\right| B_{j}\right\rangle=i F f_{6 j k}+D d_{6 j k}
$$

where $f_{i j k}$ and $d_{i j k}$ are the $\mathrm{SU}(3)$ coefficients and $F$ and $D$ the reduced matrix elements.

With the use of these soft-meson techniques and the SU(3) symmetry one can now relate the physical amplitudes of the nonleptonic hyperon decays into a pion plus a nucleon or a hyperon, $B \rightarrow B^{\prime}+\pi$, with the unphysical amplitudes of the other members of the meson octet, the kaon, and the $\eta$. One obtains relations such as [42]

$$
\begin{gathered}
\left\langle n K^{0}\left|H_{\mathrm{PV}}\right| n\right\rangle=\sqrt{\frac{3}{2}} \Lambda_{-}^{0}-\frac{1}{\sqrt{2}} \Sigma_{0}^{+}, \\
\left\langle p K^{0}\left|H_{\mathrm{PV}}\right| p\right\rangle=-\sqrt{2} \Sigma_{0}^{+}, \\
\left\langle n K^{+}\left|H_{\mathrm{PV}}\right| p\right\rangle=\sqrt{\frac{3}{2}} \Lambda_{-}^{0}+\frac{1}{\sqrt{2}} \Sigma_{0}^{+}, \\
\left\langle n \eta\left|H_{\mathrm{PV}}\right| \Lambda\right\rangle=\sqrt{\frac{3}{2}} \Lambda_{-}^{0},
\end{gathered}
$$

where $\Sigma_{0}^{+}$stands for $\left\langle p \pi^{0}\left|H_{\mathrm{PV}}\right| \Sigma^{+}\right\rangle$, the PV amplitude of the decay $\Sigma^{+} \rightarrow p \pi^{0}$, which is experimentally accessible. We have used the standard notation according to which the hy- 
peron and meson charges appear as superscript and subscript, respectively. We point out that using the isospin structure of the potential defined in the previous chapter, the $\left\langle N K\left|H_{\mathrm{PV}}\right| N\right\rangle$ matrix elements are connected to the coupling constants $C_{K}^{\mathrm{PV}}$ and $D_{K}^{\mathrm{PV}}$ of Eq. (29) via

$$
\begin{gathered}
\left\langle n K^{0}\left|H_{\mathrm{PV}}\right| n\right\rangle=C_{K}^{\mathrm{PV}}+D_{K}^{\mathrm{PV}}, \\
\left\langle p K^{0}\left|H_{\mathrm{PV}}\right| p\right\rangle=D_{K}^{\mathrm{PV}}, \\
\left\langle n K^{+}\left|H_{\mathrm{PV}}\right| p\right\rangle=C_{K}^{\mathrm{PV}} .
\end{gathered}
$$

As shown above, the symmetry of SU(3) allows connecting the amplitudes of the physical pionic decays with those of the unphysical decays involving $\eta$ 's and kaons. $\mathrm{SU}(6)_{w}$, on the other hand, furthermore permits relating the amplitudes involving pseudoscalar mesons with those of the vector mesons. For details we refer the reader to Refs. [18,42]; we just list here the final relations in terms of the coupling constants defined in the previous section, rather than matrix elements:

$$
\begin{gathered}
A_{\pi}=\frac{1}{\sqrt{2}} \Lambda_{-}^{0}, \\
A_{\eta}=\sqrt{\frac{3}{2}} \Lambda_{-}^{0}, \\
C_{K}^{\mathrm{PV}}=\sqrt{\frac{3}{2}} \Lambda_{-}^{0}+\frac{1}{\sqrt{2}} \Sigma_{0}^{+}, \\
D_{K}^{\mathrm{PV}}=-\sqrt{2} \Sigma_{0}^{+}, \\
A_{\rho}=\varepsilon_{\rho}=\frac{2}{3} \Lambda_{-}^{0}-\frac{1}{\sqrt{3}} \Sigma_{0}^{+}+\sqrt{3} a_{T}, \\
A_{\omega}=\varepsilon_{\omega}=\Sigma_{0}^{+}-\frac{1}{3} a_{T}, \\
D_{K^{*}}^{\mathrm{PV}}=-\frac{1}{3} \Sigma_{0}^{+}+\frac{8}{9} a_{T} . \\
{ }^{\mathrm{PV}} \Lambda_{-}^{0}+\frac{1}{3} \Sigma_{0}^{+}+\frac{10}{3} a_{T}, \\
2
\end{gathered}
$$

The numerical values of the constants are given in Table III. Note, that an additional parameter $a_{T}$ is present in the coupling constants for the vector mesons. This coupling, which is very small in the case of pion emission due to partially conserved axial current (PCAC), can be calculated in the factorization approximation where the vector meson is coupled to the vacuum by one of the weak currents. We use the numerical value of $a_{T}=-0.953 \times 10^{-7}$ from Ref. [42].

\section{B. The parity-conserving amplitudes}

A description of the physical nonleptonic decay amplitudes $B \rightarrow B^{\prime}+\pi$ can also be performed by using a lowestorder chiral analysis. Employing a chiral Lagrangian truncated at lowest order in the energy expansion for the PV (or $s$-wave) amplitudes yields results identical to those discussed above for pseudoscalar mesons. However, if one defines the lowest-order chiral Lagrangian for PC (or $p$-wave) amplitudes, one finds that such an operator has to vanish since it has the wrong transformation properties under $C P$. Thus, the only allowed chiral Lagrangian at lowest order can generate PV but not PC terms.

The standard method to compute the PC amplitudes is the so-called pole model. As shown in Ref. [44], this approach can be motivated by considering the transition amplitude for the nonleptonic emission of a meson:

$$
\begin{aligned}
& \left\langle B^{\prime} M_{i}(q)\left|H_{w}\right| B\right\rangle \\
& \quad=\int d^{4} x \mathrm{e}^{i q x} \theta\left(x^{0}\right)\left\langle B^{\prime}\left|\left[\partial A_{i}(x), H_{w}(0)\right]\right| B\right\rangle .
\end{aligned}
$$

Inserting a complete set of intermediate states $\{|n\rangle\}$ one can show that

$$
\begin{aligned}
\left\langle B^{\prime} M_{i}(q)\left|H_{w}\right| B\right\rangle= & -\int d^{3} x \mathrm{e}^{i q x}\left\langle B^{\prime}\left|\left[A_{i}^{0}(x, 0), H_{w}(0)\right]\right| B\right\rangle \\
& -q_{\mu} M_{i}^{\mu}
\end{aligned}
$$

where

$$
\begin{aligned}
M_{i}^{\mu}= & (2 \pi)^{3} \sum_{n}\left[\delta\left(\mathbf{p}_{n}-\mathbf{p}_{B^{\prime}}-\mathbf{q}\right)\right. \\
& \times \frac{\left\langle B^{\prime}\left|A_{i}^{\mu}(0)\right| n\right\rangle\left\langle n\left|H_{w}(0)\right| B\right\rangle}{p_{B}^{0}-p_{n}^{0}}+\delta\left(\mathbf{p}_{B}-\mathbf{p}_{n}-\mathbf{q}\right) \\
& \left.\times \frac{\left\langle B^{\prime}\left|H_{w}(0)\right| n\right\rangle\left\langle n\left|A_{i}^{\mu}(0)\right| B\right\rangle}{p_{B}^{0}-q^{0}-p_{n}^{0}}\right] .
\end{aligned}
$$

While the first term in Eq. (66) becomes the commutator introduced in Eq. (59), the second term contains contributions from the $\frac{1}{2}^{+}$ground-state baryons which are singular in the SU(3) soft meson limit. These pole terms become the leading contribution to the PC amplitudes. We note in passing that in principle, such baryon pole terms can also contribute to the PV amplitudes, however, more detailed studies [44] showed that their magnitude is only several per cent of the leading current algebra contribution.

We begin by computing the $p$-wave amplitude of the $\Lambda \rightarrow N \pi$ decay since here we can compare with experiment. The contribution to the PC weak vertex coming from the baryon pole diagrams shown in Figs. 3(a) and 3(b) are given by

$$
B_{\pi}=g_{N N \pi} \frac{1}{m_{\Lambda}-m_{N}} A_{N \Lambda}+g_{\Lambda \Sigma \pi} \frac{1}{m_{N}-m_{\Sigma}} A_{N \Sigma},
$$

where $A_{N \Lambda}$ and $A_{N \Sigma}$ are weak baryon $\rightarrow$ baryon transition amplitudes. These quantities can be determined via current algebra/PCAC as before:

$$
\lim _{q \rightarrow 0}\left\langle\pi^{0} n\left|H_{\mathrm{PV}}\right| \Lambda\right\rangle=\frac{-i}{F_{\pi}}\left\langle n\left|\left[F_{\pi^{0}}^{5}, H_{\mathrm{PV}}\right]\right| \Lambda\right\rangle=\frac{i}{2 F_{\pi}}\left\langle n\left|H_{\mathrm{PC}}\right| \Lambda\right\rangle,
$$




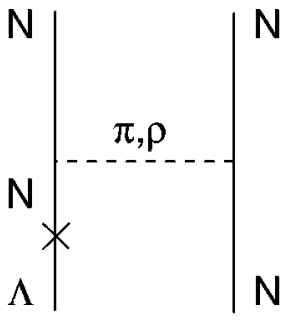

(a)

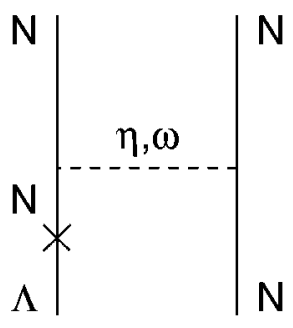

(c)

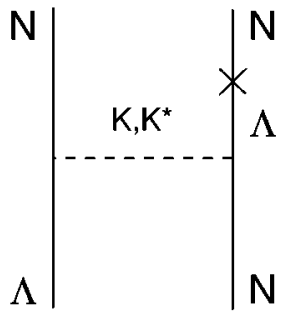

(e)

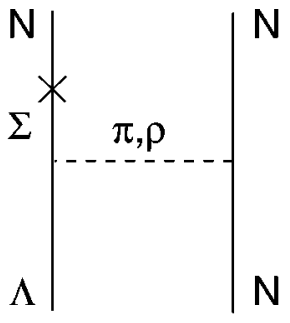

(b)
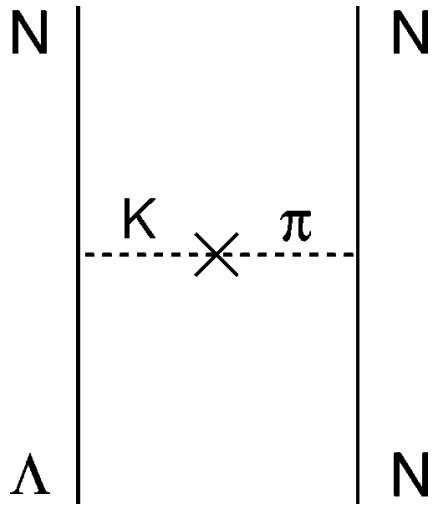

FIG. 4. Meson pole diagram contributing to the $\Lambda N \rightarrow N N$ transition amplitude.

For the $\eta$ contribution the PC $\Lambda N \eta$ term, shown in Figs. $3(\mathrm{c})$ and $3(\mathrm{~d})$, can be written as

$$
B_{\eta}=g_{N N \eta} \frac{1}{m_{\Lambda}-m_{N}} A_{N \Lambda}+g_{\Lambda \Lambda} \eta \frac{1}{m_{N}-m_{\Lambda}} A_{N \Lambda}
$$

while for the kaon [Figs. 3(e) and 3(f)], the expressions are

$$
\begin{aligned}
\left\langle n K^{+}\left|H_{\mathrm{PC}}\right| p\right\rangle= & C_{K}^{\mathrm{PC}}=g_{\Lambda p K^{+}} \frac{1}{m_{n}-m_{\Lambda}} A_{n \Lambda} \\
& +g_{p \Sigma^{0} K^{+}} \frac{1}{m_{n}-m_{\Sigma}^{0}} A_{n \Sigma} 0 \\
= & g_{\Lambda N K} \frac{1}{m_{N}-m_{\Lambda}} A_{N \Lambda}-g_{N \Sigma K} \frac{1}{m_{N}-m_{\Sigma}} A_{N \Sigma},
\end{aligned}
$$

$\Lambda$

N
FIG. 3. Baryon pole diagrams contributing to the PC weak vertices in the $\Lambda N \rightarrow N N$ transition amplitude.

$$
\begin{aligned}
\lim _{q \rightarrow 0}\left\langle\pi^{0} p\left|H_{\mathrm{PV}}\right| \Sigma^{+}\right\rangle & =\frac{-i}{F_{\pi}}\left\langle p\left|\left[F_{\pi^{0}}^{5}, H_{\mathrm{PV}}\right]\right| \Sigma^{+}\right\rangle \\
& =\frac{i}{2 F_{\pi}}\left\langle p\left|H_{\mathrm{PC}}\right| \Sigma^{+}\right\rangle .
\end{aligned}
$$

Then assuming no momentum dependence for the baryon $S$-wave decay amplitude and absorbing the $i$ factor in the definitions of $A_{N \Lambda}$ and $A_{N \Sigma}$, we obtain

$$
\begin{aligned}
A_{N \Lambda} & =i\left\langle n\left|H_{\mathrm{PC}}\right| \Lambda\right\rangle=2 F_{\pi}\left\langle\pi^{0} n\left|H_{\mathrm{PV}}\right| \Lambda\right\rangle \\
& =-\sqrt{2} F_{\pi}\left\langle\pi^{-} p\left|H_{\mathrm{PV}}\right| \Lambda\right\rangle \\
& =-4.32 \times 10^{-5} \mathrm{MeV}, \\
A_{N \Sigma}=\frac{i}{\sqrt{2}}\left\langle p\left|H_{\mathrm{PC}}\right| \Sigma^{+}\right\rangle & =\sqrt{2} F_{\pi}\left\langle\pi^{0} p\left|H_{\mathrm{PV}}\right| \Sigma^{+}\right\rangle \\
& =-4.35 \times 10^{-5} \mathrm{MeV} .
\end{aligned}
$$

$$
\begin{aligned}
\left\langle p K^{0}\left|H_{\mathrm{PC}}\right| p\right\rangle & =D_{K}^{\mathrm{PC}}=g_{p \Sigma}{ }^{+} K^{0} \frac{1}{m_{p}-m_{\Sigma}^{+}} A_{p \Sigma^{+}} \\
& =2 g_{N \Sigma K} \frac{1}{m_{N}-m_{\Sigma}} A_{N \Sigma},
\end{aligned}
$$

where we have used the relations $A_{n \Sigma^{0}}=-A_{N \Sigma}$ and $A_{p \Sigma^{+}}=\sqrt{2} A_{N \Sigma}$.

The expressions for the vector mesons are similar:

$$
\begin{gathered}
\alpha_{\rho}=g_{N N \rho}^{V} \frac{1}{m_{\Lambda}-m_{N}} A_{N \Lambda}+g_{\Lambda \Sigma}^{V} \frac{1}{m_{N}-m_{\Sigma}} A_{N \Sigma}, \\
\alpha_{\omega}=g_{N N \omega}^{V} \frac{1}{m_{\Lambda}-m_{N}} A_{N \Lambda}+g_{\Lambda \Lambda \omega}^{V} \frac{1}{m_{N}-m_{\Lambda}} A_{N \Lambda}, \\
C_{K^{*}}^{\mathrm{PC}, V}=g_{\Lambda N K^{*}}^{V} \frac{1}{m_{N}-m_{\Lambda}} A_{N \Lambda}-g_{N \Sigma K^{*}}^{V} \frac{1}{m_{N}-m_{\Sigma}} A_{N \Sigma}, \\
D_{K^{*}}^{\mathrm{PC}, V}=2 g_{N \Sigma K^{*}}^{V} \frac{1}{m_{N}-m_{\Sigma}} A_{N \Sigma},
\end{gathered}
$$

and the tensor coupling constants $\beta_{\rho}, \beta_{\omega}, C_{K^{*}}^{\mathrm{PC}, T}$, and $D_{K^{*}}^{\mathrm{PC}, T}$ are obtained from the previous expressions by replac- 
TABLE IV. $\pi$ exchange contribution to the $\Lambda N \rightarrow N N$ decay rate of ${ }_{\Lambda}^{12} \mathrm{C}$.

\begin{tabular}{lcccccc}
\hline \hline & Free & SRC & SRC+FF & \multicolumn{3}{c}{ SRC+FF+FSI } \\
& & & $\begin{array}{c}\text { Phenom. } \\
\text { Eq. }(21)\end{array}$ & Nijm93 & Reid93 \\
\hline$C(S S)$ & 0.282 & $3.4 \times 10^{-3}$ & $1.3 \times 10^{-2}$ & $4.2 \times 10^{-3}$ & $3.3 \times 10^{-3}$ & $4.0 \times 10^{-3}$ \\
$T$ & 0.858 & 0.781 & 0.637 & 0.685 & 0.566 & 0.579 \\
$\mathrm{PC}$ & 1.140 & 0.785 & 0.650 & 0.689 & 0.531 & 0.547 \\
$\mathrm{PV}$ & 0.542 & 0.447 & 0.389 & 0.421 & 0.353 & 0.345 \\
$\Gamma / \Gamma_{\Lambda}$ & 1.682 & 1.232 & 1.038 & 1.110 & 0.885 & 0.892 \\
$\Gamma_{n} / \Gamma_{p}$ & 0.182 & 0.113 & 0.120 & 0.118 & 0.104 & 0.100 \\
$\mathrm{PV} / \mathrm{PC}$ & 0.476 & 0.570 & 0.598 & 0.610 & 0.665 & 0.631 \\
$a_{\Lambda}$ & & & & & & -0.238 \\
\hline \hline
\end{tabular}

ing the strong vector couplings with the tensor ones. The numerical values of all these coupling constants can be found in Table III.

We point out that some studies have included meson pole diagrams of the form shown in Fig. 4. The contribution of these diagrams would be given by

$$
g_{\Lambda N K} \frac{1}{m_{K}^{2}-m_{\pi}^{2}} A_{K \pi},
$$

where the meson $\rightarrow$ meson weak transition amplitude $A_{K \pi}$ can also be related via PCAC to the experimental amplitude for $K \rightarrow \pi \pi$ decay, yielding $A_{K \pi}=-2.5 \times 10^{-3} \mathrm{MeV}^{2}$ [44]. There is considerable uncertainty regarding the phase between the meson and the baryon poles which lead some studies to adjust it to better reproduce the data. It has been argued [44] that the presence of these meson pole diagrams is important to fulfil the requirements of the so-called FeinbergKabir-Weinberg theorem in the nonleptonic decays. On the other hand, counting powers of energy in a chiral analysis, one finds that while the baryon pole terms are of order $q^{-1}$ the meson poles enter at next order, along with higher-order chiral Lagrangians. In general, we found these contributions to be very small and have therefore neglected them in the following. In principle, $\mathrm{SU}(6)_{w}$ can be used as well to relate the weak meson $\rightarrow$ meson pseudoscalar transition amplitudes with those of the vector mesons. The results, given here for completeness, are

$$
\begin{gathered}
A_{K \eta}=-\frac{1}{\sqrt{3}} A_{K \pi}, \\
A_{K * \rho}=A_{K \pi}, \\
A_{K * \omega}=-\frac{1}{\sqrt{3}} A_{K \pi} .
\end{gathered}
$$

\section{RESULTS}

A. $\pi$ exchange

We begin our discussion by presenting the results using only the OPE part of the weak $\Lambda N \rightarrow N N$ interaction. On one hand we would expect this meson to adequately describe at the least the long-range part of the transition potential, while on the other hand its contribution has minimal uncertainties since the weak $\Lambda N \pi$ vertex is experimentally known. It is therefore a good starting point to assess the significance of form factors as well as initial and final state correlations before including the other mesons in the potential. The results of our calculations with OPE only are shown in Table IV where the nonmesonic decay rate of ${ }_{\Lambda}^{12} \mathrm{C}$ is given in units of the free lambda decay rate $\left(\Gamma_{\Lambda}\right)$. The uncorrelated results (Free) are compared with computations that include initial $\Lambda N$ short-range correlations (SRC), form factors (FF), and final-state interactions (FSI) separately for the central $(C)$, tensor $(T)$ [adding to a total parity-conserving (PC) contribution], and parity-violating (PV) potentials. The free central term is reduced dramatically by the initial SRC, however, most of the uncorrelated central potential contribution is in fact due to the $\delta$ function in the transition potential which is completely eliminated by SRC. Without the $\delta$ function, the central part is reduced by about a factor of 2 . Including SRC, $\mathrm{FF}$, and FSI gives a negligible central decay rate. In contrast, the contribution of the tensor interaction is reduced only $10 \%$ by SRC and by $20-35 \%$ once FF and FSI are included as well. Therefore, the contribution of the central term amounts to less than $0.5 \%$ of the total $\pi$-exchange rate. This behavior has been found and discussed by other authors as well $[15,17,39]$. On the other hand, our PV potential yields about $40 \%$ of the $\pi$-exchange rate, at variance with older nuclear matter results that reported either a $15 \%$ [17] or a negligible [15] PV contribution to the rate. The total one pion exchange contribution to the nonmesonic decay rate of ${ }_{\Lambda}^{12} \mathrm{C}$ is $0.9-1.1$ $\Gamma_{\Lambda}$, depending on the choice for FSI, which is a factor $1.5-$ 2 smaller than the free value.

In previous papers $[27,28]$ we have demonstrated the sensitivity of the calculated decay rates to the form of the initial SRC. In particular, we found that older calculations using a 


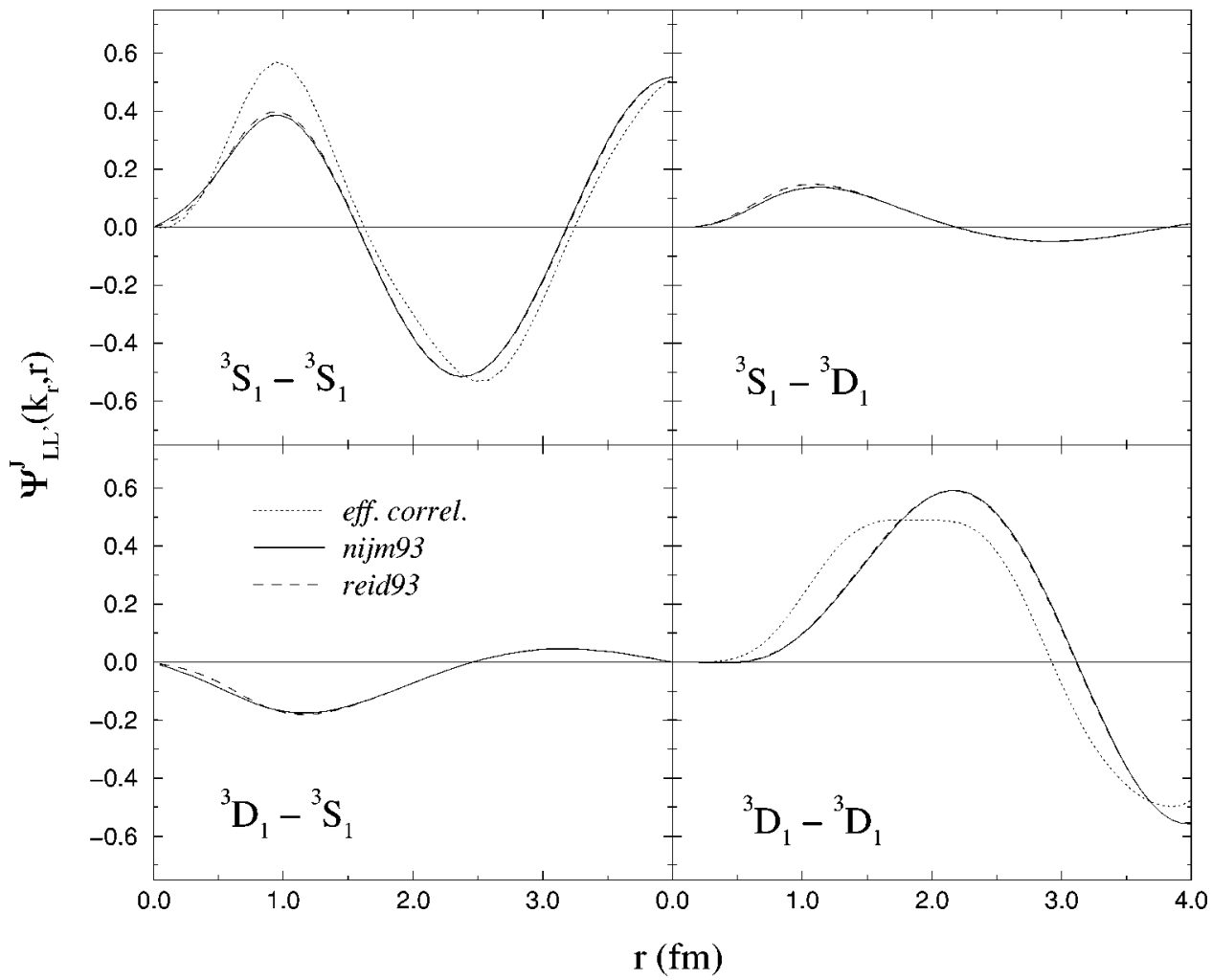

FIG. 5. ${ }^{3} S_{1}-{ }^{3} D_{1}$ coupled channel $N N$ wave functions for a relative momentum of $k_{r}$ $=1.97 \mathrm{fm}^{-1}$, obtained with the Nijmegen93 (solid line) and the Reid93 (dashed line) interactions. The dotted line represents the phenomenological correlated wave function discussed in the text.

phenomenological $N N$ correlation function $[15,20]$ to simulate $\Lambda N \mathrm{SRC}$ in the initial state, rather than SRC based on a realistic $Y N$ meson-exchange potential as is done here, tend to overpredict the amount of initial correlations. We find similar results for the final-state interactions. As shown in Table IV, the PV and PC rates are reduced by about 10 and $18 \%$, respectively, when FSI are included via a correlation function based on a realistic $N N$ potential (last two col- umns), rather than the 8 and $6 \%$ increase obtained with the phenomenological $N N$ correlation function of Eq. (20). It is comforting to see that the variation between different realistic $N N$ interactions, such as the soft-core Nijmegen and a modern version of the Reid potential, plays essentially no role. This behavior can also be understood from Figs. 5 and 6 , where we compare the different correlated wave functions for several channels and a relative momentum of $k_{r}=1.97$

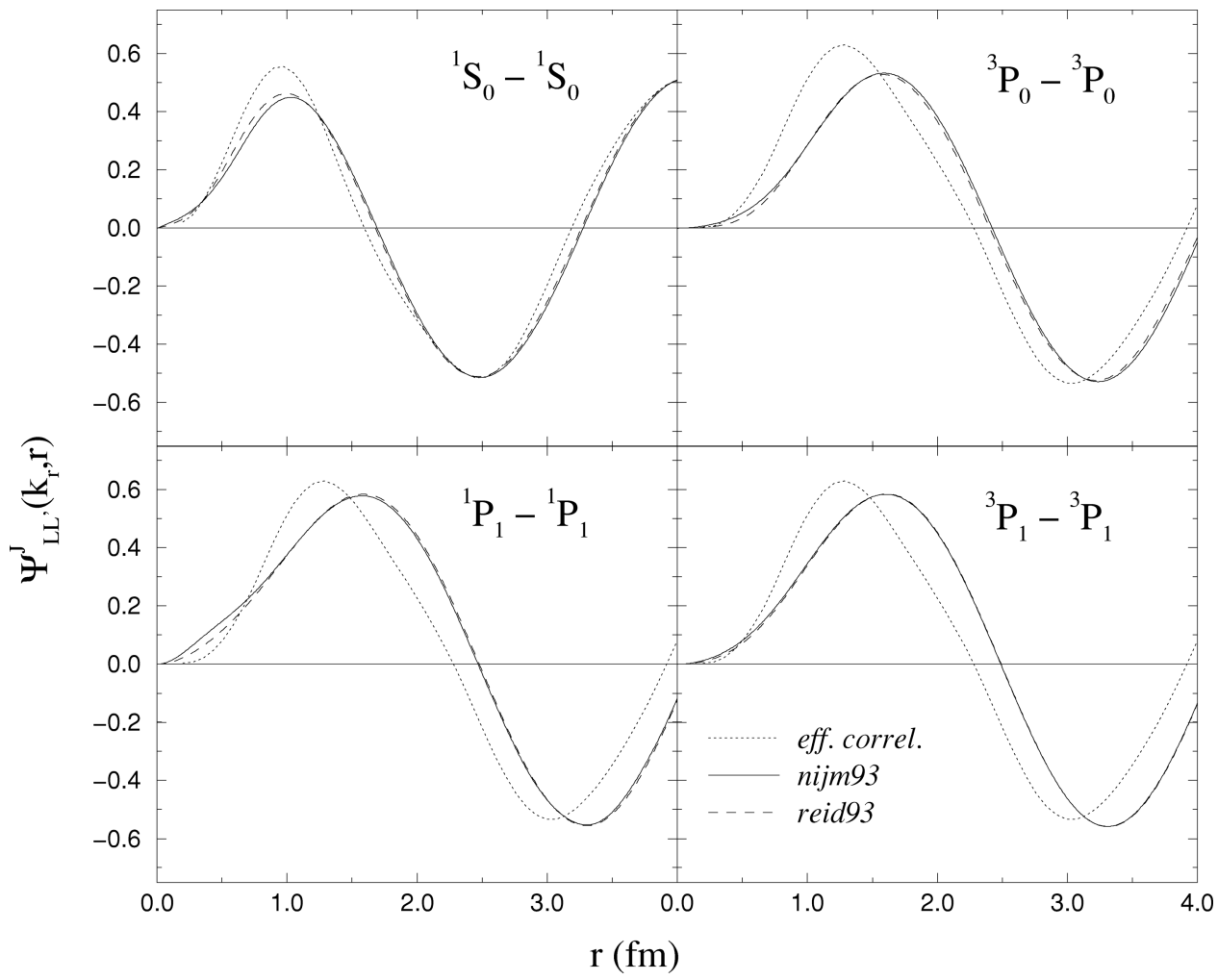

FIG. 6. Same as Fig. 5 for the uncoupled ${ }^{1} S_{0},{ }^{3} P_{0},{ }^{1} P_{1}$, and ${ }^{3} P_{1}$ channels. 
$\mathrm{fm}^{-1}$. The figures demonstrate that the phenomenological correlated wave function overestimates the realistic $N N$ wave functions at intermediate distances $(0.5-1.5 \mathrm{fm})$ while it underestimates them at short distances. As a result, the phenomenological approach overestimates the decay rate by about $20 \%$. Including realistic final state correlations leads to an interference between central and tensor transitions. For phenomenological FSI the central and tensor contributions to the total rate can be seen in Table IV to add incoherently. Once a realistic $N N$ potential is used this incoherence is replaced by destructive interference. While this is only a small effect for the pion due to the small size of the central potential term this interference is more significant for the vector mesons where the central transition amplitude is large compared to the tensor term.

The second quantity of interest displayed in Table IV which is sensitive to the isospin structure of the transition amplitude is the neutron-to-proton-induced ratio $\Gamma_{n} / \Gamma_{p}$. Noticeable is the smallness of the ratio which is due to the Pauli Principle that suppresses the final $T=1, L=2, S=1$ state with its antisymmetrization factor $\left[1-(-1)^{L+S+T}\right]$. That excludes the tensor transition in the neutron-induced rate, which gives rise to $n n(T=1)$ pairs, and it is precisely this tensor piece which constitutes the largest part of the OPE diagram. However, this argument holds only for relative $\Lambda N S$ states. For nucleons in the $p$ shell there exists a relative $\Lambda N P$ state which contributes a small but nonzero amount of the tensor potential to the neutron-induced decay. Note that including initial SRC, FF, and realistic FSI reduces the $\Gamma_{n} / \Gamma_{p}$ further by about $40 \%$. This is due to the elimination of the central potential for which we obtain a value for $\Gamma_{n} / \Gamma_{p}$ of about $1 / 3$. In principle, one would expect $\Gamma_{n} / \Gamma_{p}$ $=1 / 2$ for the central term due to the statistical factor of $1 / 2$ that accounts for two identical particles in the final state. For ${ }_{\Lambda}^{12} \mathrm{C}$ this number becomes $1 / 2.4$ since we have five neutrons and six protons. The remaining difference comes from different ${ }^{1} S_{0}(T=1)$ and ${ }^{3} S_{1}(T=0)$ final state wave functions which enter the various spin-isospin channels and, therefore, lead to slightly different $\Lambda n \rightarrow n n$ and $\Lambda p \rightarrow n p$ transition amplitudes.

The suppression of the central potential term due to SRC, $\mathrm{FF}$, and FSI also explains the difference between the uncorrelated and the fully correlated ratio of PV to PC amplitudes, $\mathrm{PV} / \mathrm{PC}$, shown in Table IV as well. More relevant than this ratio, however, is the asymmetry parameter $a_{\Lambda}$ defined in Eq. (9). This quantity, which measures the interference between the PC and the PV part of the amplitude, can be accessed experimentally in contrast to the $\mathrm{PV} / \mathrm{PC}$ ratio which is merely of theoretical interest. We find that this asymmetry parameter is only mildly sensitive to initial SRC and FF but changes by more than a factor of 2 when realistic FSI are included. This observable thus clearly demonstrates that for its accurate prediction the use of a realistic $N N$ potential to describe the interactions in the final state is imperative. Below we will use realistic FSI generated with the Nijmegen potential for all results that include final state correlations.

Comparing our results obtained here with older nuclear matter computations $[14,15,17,18]$ we point out that the fully correlated total rate in nuclear matter is predicted to be in the range of 1.85-2.3, thus overpredicting our shell model calculations by more than a factor of 2 . When a local density approximation (LDA) is performed $[4,14]$ the rate reduces to $\Gamma_{n m}=1.45 \Gamma_{\Lambda}$ for ${ }_{\Lambda}^{12} \mathrm{C}$. Although this value is further reduced when the same $\Lambda$ wave function as in the present work is used, the LDA result is still larger by about 20-40\%, depending on the choice of the Landau-Migdal parameter which measures the initial state correlations. The remaining difference may not be entirely surprising since the LDA is expected to work well for heavier nuclei and begins to break down for $s$ - and $p$-shell nuclei. We also find that our PV potential yields about $40 \%$ of the $\pi$-exchange rate, at variance with nuclear matter results that reported negligible PV rates [15].

We conclude our discussion of the OPE only by assessing the role of the relative $\Lambda N P$-state contributions. In shell model calculations such terms naturally arise for nucleons in $p$ shell and higher orbitals when one transforms from shell model coordinates to the relative $\Lambda N$ two-body system. For $s$-shell nucleons, where one has an $S$ wave in both the relative and center-of-mass (c.m.) system motions, the transition amplitude gives a maximum contribution at the back-to-back kinematics $\mathbf{k}_{1}=-\mathbf{k}_{2}$, yielding a total c.m. system momentum $\mathbf{K}=0$. For $p$-shell nucleons one would expect the contribution from the initial relative $\Lambda N S$ state to be suppressed compared to that of the relative $P$ state since the c.m. system harmonic oscillator wave function is then a $P$ state and thus zero at $\mathbf{K}=0$. Surprisingly, we find that after integrating over all kinematics with $\mathbf{k}_{1} \neq-\mathbf{k}_{2}$, this relative $L=0$ term contributes about $90 \%$ to the $p$-shell rate [19]. Thus, once the whole phase space is included, most of the total decay rate of $p$-shell nucleons still comes from the relative $\Lambda N S$ state. Furthermore, neglecting the relative $P$-state contribution leaves the ratio $\Gamma_{n} / \Gamma_{p}$ unaltered while the asymmetry parameter $a_{\Lambda}$ is reduced by $10 \%$.

\section{B. $\pi$ and $\rho$ exchange}

In this section we begin examining the role of additional mesons. As discussed above, one is faced with the immediate difficulty that none of the weak couplings involving heavier mesons can be accessed experimentally. Thus, one is required to resort to models which in this case involve considerable uncertainty. Table $\mathrm{V}$ presents our results for the $\rho$-meson exchange alone as well as for the $\pi$ and $\rho$ exchanges combined. Since both the $\Lambda N \pi$ and the $\Lambda \mathrm{N} \rho$ couplings are obtained within the same model there is no sign ambiguity. As noted before, the central potential can now be divided into a spin-independent $(C)$ and a spin-dependent $(S S)$ piece, which are shown separately. In contrast to the pion case, the factor $m_{\rho}^{2}$ in front of the Yukawa function in the $S S$ central part of Eq. (46) enhances this contribution which then becomes comparable in magnitude to the piece containing the $\delta$ function. The two terms interfere destructively and yield a SS central part that is about half the size of the tensor contribution. In Ref. [34] we noted that SRC reduce both the central $C$ and the $S S$ part of the $\rho$-meson contribution without a $\delta$ function by almost a factor of 10 , compared to a factor of 2 in the $\pi$ case, reflecting the much shorter range of the $\rho$-exchange diagram. Similarly, the tensor interaction of the $\rho$ is reduced by a factor of 2.5 , compared to a $10 \%$ reduction in the $\pi$ case, as soon as SRC are included. The additional inclusion of FF and FSI further re- 
TABLE V. $\pi$ and $\rho$ exchange contribution to the $\Lambda N \rightarrow N N$ decay rate of ${ }_{\Lambda}^{12} \mathrm{C}$. The values in parentheses have been calculated using the weak-coupling constants by Nardulli [16].

\begin{tabular}{lccc}
\hline \hline & $\pi$ & $\rho$ & $\pi+\rho$ \\
\hline$C(C)$ & & $0.020(0.019)$ & $0.020(0.019)$ \\
$C(S S)$ & 0.003 & $0.014(0.016)$ & $0.006(0.007)$ \\
$C$ (total) & 0.003 & $0.045(0.047)$ & $0.028(0.030)$ \\
$T$ & 0.566 & $0.027(0.032)$ & $0.373(0.358)$ \\
$\mathrm{PC}$ & 0.531 & $0.021(0.023)$ & $0.445(0.428)$ \\
$\mathrm{PV}$ & 0.353 & $0.008(0.096)$ & $0.414(0.635)$ \\
$\Gamma / \Gamma_{\Lambda}$ & 0.885 & $0.029(0.120)$ & $0.859(1.063)$ \\
$\Gamma_{n} / \Gamma_{p}$ & & $0.076(0.097)$ & $0.095(0.063)$ \\
$a_{\Lambda}$ & 0.104 & $0.036(0.046)$ & $-0.100(-0.008)$ \\
\hline \hline
\end{tabular}

duces both the central and tensor rates by substantial amounts. As is evident from Table $\mathrm{V}$, the final result of the central $\rho$ contribution exceeds the $\rho$ tensor term by almost a factor of 2. Due to the strong destructive interference induced by the FSI between the tensor and the central part the total $\mathrm{PC}$ rate turns out to be smaller than either term alone.

In terms of the combined $\pi$ and $\rho$ contribution we find destructive interference between the two mesons for the PC rate but constructive interference for the PV decay mode. While the $\pi$-only PC rate is reduced by $16 \%$ when the $\rho$ is added, the $\pi$-only PV rate is enhanced by about $17 \%$ even though the $\rho$-only PV rate is very small. These counterbalancing interferences lead to a combined $\pi+\rho$ total decay rate that is very similar to that of the $\pi$ alone. The neutron to proton induced ratio $\Gamma_{n} / \Gamma_{p}$, on the other hand, is slightly decreased. This could be, in principle, surprising since the tensor rate of the $\rho$ exchange is not as dominant as it is in the $\pi$ case. As noted in Ref. [34], this is due to an interference pattern of the central $C$ and $S S$ amplitudes which is destructive for the $n$-induced and constructive for the $p$ induced mechanisms. This yields a central rate which is basically $p$ induced. The strongest change can be seen in the asymmetry parameter $a_{\Lambda}$ which is reduced by more than a factor of 2 . This reduction can be traced to the above mentioned interference pattern between the PV and PC rates which are measured by this observable.

The $\rho$ meson was the first of the heavier meson which was included in several earlier calculations. The first attempt was due to McKellar and Gibson [15] in a nuclear matter framework which evaluated the weak $\Lambda N \rho$ couplings using $\mathrm{SU}(6)$ and, alternatively, a factorization model. In their approach, which neglected the PV couplings, the phase between the $\pi$ and $\rho$ amplitudes was not determined and their final results varied dramatically with their different models for the weak coupling constants. In a more recent calculation [16], Nardulli obtains the PC couplings in a pole model approach similar to ours. Besides the ground-state baryon pole he includes the $\frac{1}{2} *$ baryon resonance pole terms as well as $K^{*}$ pole contributions that appear in Refs. $[18,42]$ but have been omitted here. The weak baryon-baryon transition amplitudes for the resonance poles are taken from a pole model analysis of hyperon $\mathrm{PC}$ pion decays which uses an $F / D$ ratio of -1 for the weak baryon transition amplitudes and adjusts the overall coupling to the experimentally measured $p$-wave $\pi$ decay rates. As pointed out in Ref. [45] the most serious problem with this fit is that it employs a $K \rightarrow \pi$ weak transition amplitude in its $K$ pole graphs that is about an order of magnitude larger than the strength extracted from the weak kaon decay mode $K \rightarrow \pi \pi$ [44]. The $K^{*}$ pole contributions are calculated using a simplified factorization approach in which a number of terms are neglected [45]. The PV couplings of Ref. [16] are computed in a pole model approach that includes baryon resonance poles with negative parity, belonging to the $\left(70,1^{-}\right)$multiplet of $\mathrm{SU}(6)$. In order to obtain the weak baryon transition amplitudes the experimental hyperon $s$-wave $\pi$ decays are used as input. Therefore, his approach for the PV weak $\Lambda N \rho$ vertex is considerably different from the analysis used in this study. For the sake of comparison, Table V also lists the $\rho$ term calculated with Nardulli's weak-coupling constants. The PC transition potentials turn out to be very similar in magnitude while the PV rate is larger by more than a factor of 10 for the $\rho$ alone. This increase enhances the $\pi+\rho$ total decay rate by about $25 \%$, while the $\Gamma_{n} / \Gamma_{p}$ and $a_{\Lambda}$ are reduced by roughly the same amount. One should point out that the close agreement in the PC terms is fortuitous since the baryon resonance pole terms which are not present in our approach contribute about $30 \%$ to the weak $\Lambda N \rho$ tensor coupling of Ref. [16]. From this comparison it becomes obvious that there is considerable uncertainty in the determination of the weak vector meson vertices.

\section{C. $K$ and $K^{*}$ exchange}

The results of our calculations for the exchanges of the strange mesons $K$ and $K^{*}$ are shown in Table VI. The kaon is the lightest meson after the pion with a strong coupling constant $g_{\Lambda N K}$ of comparable magnitude to $g_{N N \pi}$ but of opposite sign. The total decay rate for the $K$-only exchange diagram amounts to about $15 \%$ of the $\pi$ term which is the largest contribution among all the heavier mesons. As discussed further below the kaon therefore significantly interferes with the $\pi$-only rate. 
TABLE VI. $K$ and $K^{*}$ exchange contribution to the $\Lambda N \rightarrow N N$ decay rate of ${ }_{\Lambda}^{12} \mathrm{C}$. The values in parentheses have been calculated using the $N N K$ weak-coupling constants obtained when including one-loop corrections to the leading order in $\chi \mathrm{PT}[46]$.

\begin{tabular}{lccc}
\hline \hline & $K$ & $K^{*}$ & $K+K^{*}$ \\
\hline$C(C)$ & & 0.019 & $0.019(0.019)$ \\
$C(S S)$ & $0.004(0.002)$ & 0.092 & $0.130(0.122)$ \\
$C$ (total) & $0.004(0.002)$ & 0.038 & $0.063(0.058)$ \\
$T$ & $0.083(0.038)$ & 0.038 & $0.015(0.005)$ \\
$\mathrm{PC}$ & $0.093(0.044)$ & 0.037 & $0.082(0.050)$ \\
$\mathrm{PV}$ & $0.040(0.018)$ & 0.023 & $0.091(0.061)$ \\
$\Gamma / \Gamma_{\Lambda}$ & $0.133(0.062)$ & 0.060 & $0.173(0.111)$ \\
$\Gamma_{n} / \Gamma_{p}$ & $0.263(0.272)$ & 0.500 & $0.647(0.760)$ \\
$a_{\Lambda}$ & $-0.080(-0.090)$ & -0.192 & $-0.426(-0.532)$ \\
\hline \hline
\end{tabular}

In contrast to the $\pi$ and the $\rho$ which are isovector mesons and the $\eta$ and $\omega$ which are isoscalar, the $K$ and $K^{*}$ lead to both $T=0$ and $T=1 \quad N K$ coupled states and, therefore, have two independent couplings $C_{K}$ and $D_{K}$ [see Eq. (29)]. Due to their isospin structure it was pointed out several years ago [47] that including the kaon exchange has the potential to strongly influence the $\Gamma_{n} / \Gamma_{p}$ ratio. Using a simple schematic model that ignores the spin structure it was shown that the ratio $\Gamma_{n} / \Gamma_{p}$ could be estimated with the expression

$$
\frac{\Gamma_{n}}{\Gamma_{p}}=\left|\frac{A_{0}+A_{1}}{A_{0}-3 A_{1}}\right|^{2},
$$

where $A_{0}$ and $A_{1}$ are the isoscalar and isovector coupling constants, respectively, which in our case are given by

$$
\begin{gathered}
A_{0}=\frac{C_{K}}{2}+D_{K}, \\
A_{1}=\frac{C_{K}}{2} .
\end{gathered}
$$

With the PV values of Table III, we obtain $\Gamma_{n} / \Gamma_{p}=4.6$ using Eq. (80), confirming the result quoted in Ref. [47]. However, we obtain $\Gamma_{n} / \Gamma_{p}=0.23$ when the PC coupling constants of Table III are used. Our complete result, which considers the spin structure and includes both PC and PV amplitudes, turns out to be $\Gamma_{n} / \Gamma_{p}=0.26$ as shown in Table VI. This much smaller result suggests that one cannot draw conclusions about the ability of the strange mesons to drastically increase the neutron to proton ratio. Moreover, we have calculated this ratio using PV and PC amplitudes only but retaining the spin dependence and obtain, respectively, the values 1.69 and 0.03 , far away from the estimates made above using Eq. (80). Note that, as shown by Dalitz [12], the $\Delta I=1 / 2$ rule requires the ratio $\Gamma_{n} / \Gamma_{p}$ to be smaller than 2 for any meson exchange.

As can be seen in Table VI, the $\Gamma_{n} / \Gamma_{p}$ ratio for the kaon only is larger than the corresponding value for the pion by a factor of 2.5, while the asymmetry parameter obtained is very small. As discussed in the previous section our frame- work for the weak baryon-baryon-meson coupling constants assumes the validity of SU(3) [and SU(6) in the case of the vector mesons]. No attention has been paid to the effects of $\mathrm{SU}(3)$ symmetry breaking which is known to be of the order of $30 \%$. These effects have been addressed in a recent work by Savage and Springer [46] in the framework of chiral pertubation theory $(\chi \mathrm{PT})$ including one-loop corrections to the leading order. They point out that understanding the weak $N N K$ vertex could elucidate a problem regarding the nonleptonic $\pi$ decay of free hyperons. While the PV ( $s$ wave) amplitudes of these $\Delta S=1$ decays are adequately reproduced at tree level, the corresponding PC ( $p$-wave) amplitudes cannot be well described using coupling constants from the $s$ waves as input. A one-loop calculation of the leading SU(3) corrections [48], performed in $\chi \mathrm{PT}$, found that these loop corrections can change the tree level prediction of the $p$-wave amplitudes by a disturbing $100 \%$, thus raising questions about the validity of $\chi \mathrm{PT}$ in this sector. As an alternative it was suggested [48] that large cancellations may occur between tree-level $p$-wave $\pi$ decay amplitudes which would magnify the SU(3) breaking effects. The one-loop corrections to the weak $N N K$ vertex found in Ref. [46], on the other hand, modify the tree-level $p$-wave amplitudes by only up to $30 \%$. If an experimental signature for these SU(3) corrections could be found in the nonmesonic decay it would provide insight into the applicability of $\chi \mathrm{PT}$ to these reactions. Table VI shows the results of our calculations performed with the Savage-Springer weak $N N K$ couplings. As expected, the kaon rates are roughly a factor of two smaller since the improved constants are reduced by about $30 \%$. The values of the $\Gamma_{n} / \Gamma_{p}$ ratio and the asymmetry, on the other hand, are barely affected because all pieces of the transition amplitude are reduced by about the same amount.

The $K^{*}$ vector meson is the heaviest meson exchanged in our weak $\Lambda N \rightarrow N N$ transition potential. Nevertheless, due to its large weak $N N K^{*}$ and strong $\Lambda N K^{*}$ tensor couplings it is more important than either the $\rho$ or the $\omega$. The central and tensor potential contributions are comparable in size, however, due again to the interference generated by the realistic FSI that mixes $S$ and $D$ states, the total PC rate turns out to 
TABLE VII. $\eta$ and $\omega$ exchange contribution to the $\Lambda N \rightarrow N N$ decay rate of ${ }_{\Lambda}^{12} \mathrm{C}$.

\begin{tabular}{lccc}
\hline \hline & $\eta$ & $\omega$ & $\eta+\omega$ \\
\hline$C(C)$ & & 0.045 & 0.045 \\
$C(S S)$ & 0.001 & 0.009 & 0.016 \\
$C$ (Total) & 0.001 & 0.036 & 0.036 \\
$T$ & 0.005 & 0.004 & $1.5 \times 10^{-4}$ \\
$\mathrm{PC}$ & 0.006 & 0.024 & 0.035 \\
$\mathrm{PV}$ & 0.003 & 0.002 & 0.005 \\
$\Gamma / \Gamma_{\Lambda}$ & & & 0.041 \\
$\Gamma_{n} / \Gamma_{p}$ & 0.009 & 0.026 & 0.183 \\
$a_{\Lambda}$ & & & \\
\hline \hline
\end{tabular}

be of the same magnitude. The larger PV coupling constant yields a PV rate which is significantly greater than the corresponding rates for the $\rho$ and the $\omega$ exchange contribution. The total $K^{*}$-only decay rate is seen to be about half of the $K$-only rate but twice as large as the $\rho$ - and $\omega$-only rates. Due to the relative magnitude of the central and PC potential terms we find a $\Gamma_{n} / \Gamma_{p}$ ratio for the $K^{*}$ diagram which is 0.5 , about five times larger than that for the $\pi$-exchange ratio. This is a dramatic illustration of the difference in isospin structure between the various mesons. The $K^{*}$ diagram also exhibits more than twice the asymmetry parameter of the $K$ meson.

Coincidentally, the $K+K^{*}$ total rate is only slightly larger than the $K$-only total rate even though the interference between the $K$ and $K^{*}$ is important, as can be seen from the separate channel contributions. The PV rate is more than twice the $K$-only result and, as a consequence, the asymmetry parameter is enhanced dramatically. The $\Gamma_{n} / \Gamma_{p}$ ratio also turns to be quite large, although it remains to be seen how the interference between all the mesons actually affects the final result for the observables. This is discussed in Sec. VI E.

\section{D. $\eta$ and $\omega$ exchange}

Table VII presents our results for the isoscalar mesons alone. The $\eta$-meson exchange contribution is by far the smallest of the different mesons included in our potential. This may come as no surprise since it is known that including the $\eta$ in phase shift fits of $N N$ potentials influences the parameters only marginally. The main reason for the small size of the $\eta$ exchange lies in the magnitude of the strong $N N \eta$ coupling constant, which is not well determined but is known to be much smaller than both $g_{N N \pi}$ and $g_{\Lambda N K}$. We have used the value of the Nijmegen potential which is $g_{N N \eta}=6.4$ even though the $N N$ phase shifts are very insensitive to this coupling. Recent data on $\eta$ photoproduction on the nucleon find a much reduced value of around $g_{N N \eta}=1.4$ [49]. Should these conclusions be confirmed then clearly the $\eta$ can safely be neglected in both the strong $N N$ sector as well as the weak $\Lambda N \rightarrow N N$ transition potential discussed here. As shown in Table VII, the behavior of the $\eta$ contribution follows that of the $\pi$ term: a negligible cen- tral term, the largest piece coming from the tensor potential, and a PV rate about half the size of the tensor term. Note that in contrast to the pion there is no charge exchange term for the proton-induced decay, thus the $\Gamma_{n} / \Gamma_{p}$ for the $\eta$ only is larger than that for the pion by almost a factor of four.

The $\omega$-meson exchange provides an interesting contrast to the $\rho$ contribution since for the strong $N N \rho$ vertex the vector coupling is relatively small and the tensor coupling is large, while the reverse is true for the strong $N N \omega$ couplings. The weak PC and PV couplings (see Table III), on the other hand, are comparable in size. This pattern of strong couplings is reflected in the distribution of decay strength seen in Table VII. The largest contribution comes from the spinindependent central term which is about twice as large as the corresponding term for the $\rho$. The $\omega$ tensor term, however, is smaller than the $\rho$ tensor potential by about a factor of 7 . The interference of the various terms yield a total PC rate for the $\omega$ meson that is very similar in magnitude to the $\rho$ but generates a much larger $\Gamma_{n} / \Gamma_{p}$. Similar to the $\rho$ the PV rate is negligible due to the small size of the weak PV $\Lambda N \omega$ coupling constant. No other models are available for weak vertices involving this meson.

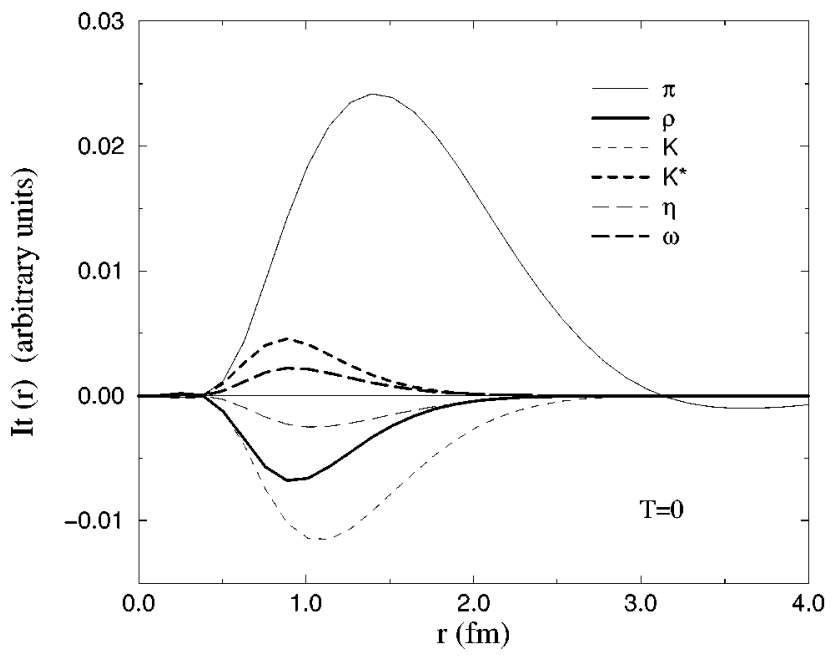

FIG. 7. Contribution of the different mesons to the integrand of the ${ }^{3} S_{1}-{ }^{3} D_{1}(T=0)$ correlated weak transition amplitude. 


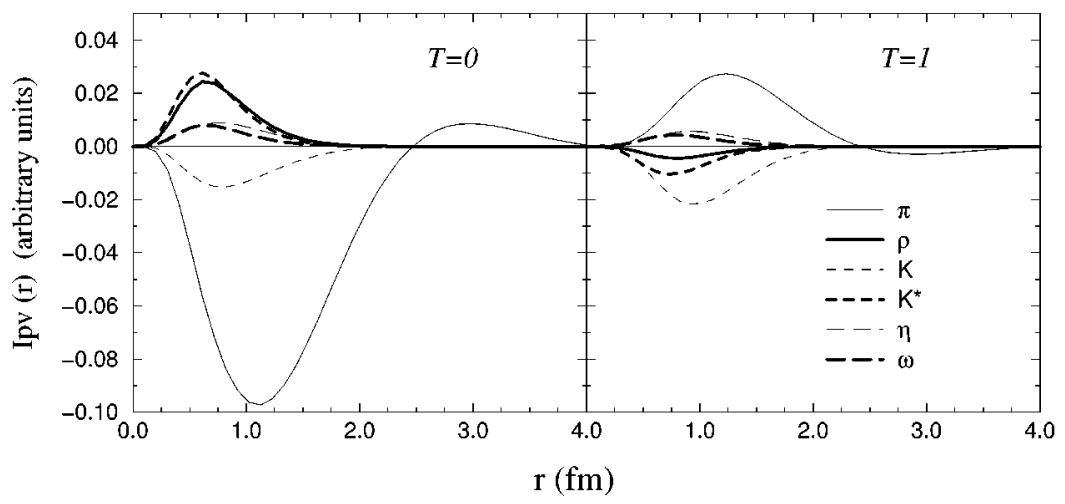

FIG. 8. Same as Fig. 7 for the PV ${ }^{3} S_{1}-{ }^{1} P_{1}$ $(T=0)$ and ${ }^{3} S_{1}-{ }^{3} P_{1}(T=1)$ transition amplitudes.

\section{E. The full weak one-meson-exchange potential}

In this subsection, we explore the effect of including all the mesons discussed before on the weak decay observables. For this purpose we show in Figs. 7 and 8 the contribution of the different mesons to the integrand of Eq. (18) for relevant transition channels. Figure 7 displays the tensor transition ${ }^{3} S_{1} \rightarrow{ }^{3} D_{1}(T=0)$ of the PC amplitudes since it yields the most important contribution for pseudoscalar mesons and gives rise to important interference effects when the contributions of the mesons are added in pairs of identical isospin. As is evident from the figure, the pion-exchange contribution dominates, not only in magnitude but also in range; a consequence of the pion being the lightest meson. As expected, the kaon provides the second-largest contribution with a range somewhat less than that of the pion, followed by the heavier mesons with an even shorter range. Note that the contribution of each isospinlike pair $\left[(\pi, \rho),\left(K, K^{*}\right),(\eta, \omega)\right]$ interferes destructively, thus the large tensor contribution of each pseudoscalar meson is partially cancelled by that of its vector meson partner, an effect that can also be explicitly seen in Tables V, VI, and VII, discussed above. We have not shown the integrands of the central transitions since they are very small for the pseudoscalar mesons.

Significant interferences are also observed for the integrands of the PV transitions ${ }^{3} S_{1} \rightarrow{ }^{1} P_{1} \quad(T=0)$ and ${ }^{3} S_{1} \rightarrow{ }^{3} P_{1}(T=1)$, shown in Fig. 8. Again, we find the pion to be dominant among the mesons in the $T=0$ transition, while the contribution of the other mesons play a more important role in the $T=1$ channel.

Below we discuss results for the different observables characteristic of the weak decay. We compare the results obtained with the Nijmegen strong-coupling constants with those obtained using the Jülich strong couplings given in Table III. Although in principle the strong couplings also affect the PC weak vertices through the pole model, our goal here is to assess, for one particular model of weak couplings, the effect of using strong-coupling constants from two different $Y N$ potentials which fit the hyperon-nucleon scattering data equally well.

The results in Table VIII again demonstrate the significance of the short-range correlations and form factors in the nonmesonic decay. Adding the heavier mesons without form factors and SRC (column free) leads to a total rate that fluctuates significantly, with the additional mesons giving an appreciable contribution to the $\pi$-exchange rate. This behavior is considerably suppressed by short-range effects, as shown in the second column. The rate is especially sensitive to the inclusion of the strange mesons. While including the $\rho$ meson has almost no effect the addition of kaon exchange reduces the total rate by almost $50 \%$ when the Nijmegen strong couplings are used. The reduction is mostly compensated by the addition of the $K^{*}$, yielding a rate $15 \%$ below the pion-only decay rate. The situation is similar for the $\eta$ and $\omega$, their combined effect on the rate is negligible. Thus, with Nijmegen couplings adding the heavier mesons gives a reduction of only $15 \%$. The situation is slightly different when the Jülich strong-coupling constants are employed; their omission of the $\eta$ and their larger $K^{*}$ and $\omega$ couplings lead to a total rate $15 \%$ larger than the pion-only rate. This indicates that the results are sensitive to the model used for the strong vertices, although both results are consistent with the present experimental values. This sensitivity to the

TABLE VIII. Free and fully correlated nonmesonic decay rate of ${ }_{\Lambda}^{12} \mathrm{C}$ in units of the free $\Lambda$ decay rate $\Gamma_{\Lambda}$. The values in parentheses have been calculated using the Jülich- $B$ coupling constants at the strong vertex.

\begin{tabular}{lcc}
\hline \hline & Free & SRC+FF+FSI \\
\hline$\pi$ & $1.682(1.682)$ & $0.885(0.885)$ \\
$+\rho$ & $2.055(2.325)$ & $0.859(0.831)$ \\
$+K$ & $1.336(1.699)$ & $0.497(0.506)$ \\
$+K^{*}$ & $2.836(3.821)$ & $0.760(0.902)$ \\
$+\eta$ & $2.467(3.821)$ & $0.683(0.902)$ \\
$+\omega$ & $2.301(4.338)$ & $0.753(1.023)$ \\
Weak $K$ couplings & & \\
from $\chi$ PT $[46]$ & & $0.844(1.104)$ \\
\hline \hline
\end{tabular}


TABLE IX. Weak decay observables for ${ }_{\Lambda}^{12} \mathrm{C}$. The values in parentheses have been calculated using the Jülich- $B$ coupling constants at the strong vertex.

\begin{tabular}{lccc}
\hline \hline & $\Gamma_{n} / \Gamma_{p}$ & $\mathrm{PV} / \mathrm{PC}$ & $a_{\Lambda}$ \\
\hline$\pi$ & $0.104(0.104)$ & $0.665(0.665)$ & $-0.238(-0.238)$ \\
$+\rho$ & $0.095(0.096)$ & $0.930(1.137)$ & $-0.100(-0.052)$ \\
$+K$ & $0.030(0.029)$ & $2.413(3.206)$ & $-0.138(-0.074)$ \\
$+K^{*}$ & $0.049(0.070)$ & $1.797(1.968)$ & $-0.182(-0.202)$ \\
$+\eta$ & $0.058(0.070)$ & $2.249(1.968)$ & $-0.200(-0.202)$ \\
$+\omega$ & $0.068(0.109)$ & $2.077(1.675)$ & $-0.316(-0.368)$ \\
Weak & & & \\
$N N K$-couplings & & & $-0.302(-0.350)$ \\
from $\chi$ PT [46] & $0.080(0.108)$ & $1.678(1.436)$ & \\
\hline \hline
\end{tabular}

strong-coupling constants is unfortunate since it will certainly complicate the task of extracting weak couplings from this reaction. Improved $Y N$ potentials which narrow the range of the strong-coupling constants are required to reduce this uncertainty. Table VIII also shows the results obtained when the $N N K$ weak-coupling constants derived with oneloop corrections to the leading order in $\chi \mathrm{PT}$ [46] are used. Due to the smaller value of the coupling constants the effect of the $K$ meson is reduced and thus the total rate is increased by about $10 \%$.

The results for the ratio of the neutron- to proton-induced partial rates $\Gamma_{n} / \Gamma_{p}$ are shown in Table IX. The neutron- to proton-induced ratio is, as expected, quite sensitive to the isospin structure of the exchanged mesons. It has been known for a long time that pion exchange alone produces only a small ratio [15]. While the role of the $\rho$ is limited it is again the inclusion of the two strange mesons that dramatically modifies this partial ratio. Including the $K$ exchange which interferes destructively with the pion amplitude in the neutron-induced channel (see, for example, the $T=1 \mathrm{PV}$ transition amplitudes of Fig. 8) leads to a reduction of the ratio by more than a factor of 3 . The $K^{*}$, on the other hand, adds contructively. Again, an indication of this behavior can be seen in Figs. 7 and 8. In the $T=1 \mathrm{PV}$ channel, relevant for the $n$-induced rate, the $K$ and $K^{*}$ amplitudes have the same sign, whereas in both $T=0$ channels the interference between the two strange mesons is destructive and, as a consequence, the $p$-induced rate is lowered with respect to the $n$-induced rate. Using the Nijmegen strong-coupling constants leads to a final ratio that is $34 \%$ smaller than the piononly ratio, while using the Jülich couplings leave this ratio unchanged, due again mostly to their larger $K^{*}$ and $\omega$ couplings. Employing the weak $N N K$ couplings calculated with $\chi$ PT we obtain an increase of the $\Gamma_{n} / \Gamma_{p}$ ratio by $17 \%$ with Nijmegen couplings while the ratio remains unchanged for the Jülich model.

Even though it is not an observable, Table IX also presents the ratio of $\mathrm{PV}$ to $\mathrm{PC}$ rates to aid in the comparison with other theoretical calculations. Again, adding the strange mesons produces the largest effect, especially when using the Jülich strong couplings. The final PV/PC ratio is larger by more than a factor of 2 compared to the pion-only ratio. The results quoted in Ref. [18] are of the order of 1 and, therefore, closer to our results obtained with the Jülich model.
The intrinsic asymmetry parameter $a_{\Lambda}$ shown in Table IX is also found to be very sensitive to the different mesons included in the model. This is the only observable which is changed dramatically by the inclusion of the $\rho$, reducing the pion-only value by more than a factor of 2 . Adding the other mesons increases $a_{\Lambda}$, leading to a result about $30 \%$ larger than for $\pi$ exchange alone in the case of the Nijmegen couplings and 50\% larger for the Jülich model. The effect of using the weak $N N K$ couplings from $\chi \mathrm{PT}$ is very small for this observable.

\section{F. Comparison with experiment}

Our final results for various hypernuclei are presented in Table X. We find an overall agreement between our results for the nonmesonic rate and the experimental values, especially when the $\chi \mathrm{PT}$ weak couplings for the $K$ meson are used, which yield somewhat larger rates.

It has been the hope for many years that the inclusion of additional mesons would dramatically increase the ratio of neutron- to proton-induced rates. Here we find the opposite to be true. The final ratio greatly underestimates the newer central experimental values, although the large experimental error bars do not permit any definite conclusions at this time. On the other hand, the proton-induced rate which has errors of the same magnitude as the total rate is overpredicted by our calculations by up to a factor of 2. It is the neutroninduced rate which has been very difficult to measure accurately. It is somewhat surprising that while both individual rates appear in disagreement with the data their sum conspires to a total rate which reproduces the measurements. Other mechanisms that have been explored to remedy this puzzle include quark-model calculations which yield a large violation of the $\Delta I=1 / 2$ rule [21,22], and the consideration of the $3 N$ emission channel $(\Lambda N N \rightarrow N N N)$ as a result of the pion being absorbed on correlated $2 N$ pairs $[3,4]$. A recent reanalysis [52], which includes FSI of the three nucleons on their way out of the nucleus via a Monte Carlo simulation, shows that the $2 N$-induced channel further increases the experimental error bars and leads to an experimental value compatible with the predictions of the OPE model. However, the same reference shows that a comparison of the calculated proton spectrum with the experimental one favors values of $\Gamma_{n} / \Gamma_{p}=2-3$. It is therefore imperative, before speculating further about the deficiencies of the present models in repro- 
TABLE X. Weak decay observables for various hypernuclei. The values in parentheses have been calculated using the $N N K$ weak-coupling constants obtained when including one-loop corrections to the leading order in $\chi$ PT [46].

\begin{tabular}{lccc}
\hline \hline & ${ }_{\Lambda}^{5} \mathrm{He}$ & ${ }_{\Lambda}^{11} \mathrm{~B}$ & ${ }_{\Lambda}^{12} \mathrm{C}$ \\
\hline$\Gamma / \Gamma_{\Lambda}$ & $0.414(0.467)$ & $0.611(0.686)$ & $0.753(0.844)$ \\
Expt. & $0.41 \pm 0.14[7]$ & $0.95 \pm 0.13 \pm 0.04[9]$ & $1.14 \pm 0.2[7]$ \\
& & & $0.89 \pm 0.15 \pm 0.03[9]$ \\
$\Gamma_{n} / \Gamma_{p}$ & $0.073(0.089)$ & $0.084(0.099)$ & $0.068(0.080)$ \\
Expt. & $0.93 \pm 0.55[7]$ & $1.04_{-0.48}^{+0.59}[7]$ & $1.33_{-0.81}^{+1.12}[7]$ \\
& & $2.16 \pm 0.58_{-0.95}^{+0.45}[9]$ & $1.87 \pm 0.59_{-1.00}^{+0.32}[9]$ \\
& & $0.70 \pm 0.3[51]$ & $0.70 \pm 0.3[51]$ \\
& & $0.52 \pm 0.16[51]$ & $0.52 \pm 0.16[51]$ \\
$\Gamma_{p} / \Gamma_{\Lambda}$ & $0.386(0.428)$ & $0.563(0.624)$ & $0.705(0.782)$ \\
Expt. & $0.21 \pm 0.07[7]$ & $0.30_{-0.11}^{+0.15}[9]$ & $0.31_{-0.11}^{+0.18}[9]$ \\
& & $-0.391(-0.378)$ & $-0.316(-0.302)$ \\
$a_{\Lambda}$ & $-0.273(-0.264)$ & $-0.120(-0.116)$ & $-0.030(-0.029)$ \\
$\mathcal{A}\left(0^{\circ}\right)$ & & $-0.20 \pm 0.10[10]$ & $-0.01 \pm 0.10[10]$ \\
Expt. & & \\
\hline \hline
\end{tabular}

ducing this ratio, to carry out more precise experiments such as the measurement of the number of protons emitted per $\Lambda$ decay, suggested in Ref. [52].

Regarding the asymmetry parameter, comparison with experiment can only be made at the level of the measured proton asymmetry. As discussed in Sec. II, this quantity is determined as a product of the asymmetry parameter $A_{p}$, characteristic of the weak decay, and the polarization of the hypernucleus $P_{y}$ which must be determined theoretically. The energy resolution of the experiment measuring the decay of polarized ${ }_{\Lambda}^{12} \mathrm{C}$ produced in a $\left(\pi^{+}, K^{+}\right)$reaction [10] was 5-7 MeV which did not allow distinguishing between the first three $1^{-}$states. Before the weak decay occurs, the two excited states decay electromagnetically to the ground state. Therefore, in order to determine the polarization at this stage, one requires (i) the polarization of the ground and excited states, together with the corresponding formation cross sections and (ii) an attenuation coefficient to account for the loss of polarization in the transition of the excited states to the ground state. In Ref. [53], hypernuclear production cross sections and polarizations have been estimated for the $\left(\pi^{+}, K^{+}\right)$reaction in the distorted wave impulse approximation with configuration-mixed wave functions. We note that the sum of the cross sections for the two excited $1^{-}$states amounts to $40 \%$ relative to the ground-state peak, which is consistent with the $(31 \pm 8) \%$ obtained in a fit to the Brookhaven ${ }^{12} \mathrm{C}\left(\pi^{+}, K^{+}\right){ }_{\Lambda}^{12} \mathrm{C}$ spectrum [54]. Using the values of Ref. [53] for the polarization and cross sections of the $1^{-}$states in ${ }_{\Lambda}^{12} \mathrm{C}$ together with the spin depolarization formalism of Ref. [55], we obtain $P_{y}=-0.19$. This value, together with $A_{p}=0.151$ (Nijmegen) or 0.175 (Jülich), determined from $a_{\Lambda}$ using Eq. (9), leads to an asymmetry $\mathcal{A}=-0.029$ (Nijmegen) or -0.033 (Jülich), which lies within the uncertainties of the experimental result.

The hypernucleus ${ }_{\Lambda}^{11} \mathrm{~B}$ is created by particle emission from excited states of ${ }_{\Lambda}^{12} \mathrm{C}$ in which a $\Lambda$ in a $p_{1 / 2}$ or $p_{3 / 2}$ orbit is coupled to a ${ }^{11} \mathrm{C}$ core in its ground state. The window of excitation energy that spans $1.55 \mathrm{MeV}$ between the $\left(p+{ }_{\Lambda}^{11} \mathrm{~B}\right)$ and the $\left(\Lambda+{ }^{11} \mathrm{C}\right)$ particle decay threshold contains three positive-parity states: two $2^{+}$states separated by $\sim$ $800 \mathrm{keV}$ and a narrow $0^{+}$state just below the $\left(\Lambda+{ }^{11} \mathrm{C}\right)$ threshold. Using the same model of Ref. [53], which predicts equal formation cross sections for the $2_{1}^{+}$and $2_{2}^{+}$states, and neglecting the $0^{+}$state because of its relatively small cross section, we obtain a polarization of $P_{y}=-0.29$. However, hypernuclear structure calculations by Auerbach et al. [56] predicted strong configuration mixing which reduced the cross section of the lower $2^{+}$state by a factor of three relative to the higher one. This prediction was verified by a reanalysis of older emulsion data [57]. Taking these relative weights into account, we obtain the value $P_{y}=-0.43$, which is the one used in Table $\mathrm{X}$ and leads to better agreement with the experimental asymmetry. Just as in the case of the proton- to neutron-induced ratio, the present level of uncertainty in the experiment does not yet permit using the asymmetry as an observable that differentiates between different models for the weak decay.

In order to avoid the need for theoretical input and access $A_{p}$ directly, a new experiment at KEK [58] is measuring the decay of polarized ${ }_{\Lambda}^{5} \mathrm{He}$, extracting both the pion asymmetry from the mesonic channel $\mathcal{A}_{\pi^{-}}$and the proton asymmetry from the nonmesonic decay $\mathcal{A}$. The asymmetry parameter $a_{\pi^{-}}$of the pionic channel has been estimated to be very similar to that of the free $\Lambda$ decay [59] and, therefore, the hypernuclear polarization can now be obtained from the relation $P_{y}=\mathcal{A}_{\pi^{-}} / a_{\pi^{-}}$. This in turn can then be used as input, together with the measured value of $\mathcal{A}$, to determine the asymmetry parameter for the nonmesonic decay from the equality $A_{p}=\mathcal{A} / P_{y}$. This experiment will not only allow a clean extraction of the nonmesonic asymmetry parameter but will also check theoretical model predictions for the amount of hypernuclear polarization. 
TABLE XI. Matrix elements of the tensor operator evaluated between generalized spherical harmonic states of definite $J, L$, and $S$.

\begin{tabular}{lccc}
\hline \hline$S_{L_{r} L^{\prime}}$ & $L^{\prime}=J+1$ & $L^{\prime}=J$ & $L^{\prime}=J-1$ \\
\hline$L_{r}=J+1$ & $\frac{-2(J+2)}{2 J+1}$ & 0 & $\frac{6 \sqrt{J(J+1)}}{2 J+1}$ \\
$L_{r}=J$ & 0 & 2 & 0 \\
$L_{r}=J-1$ & $\frac{6 \sqrt{J(J+1)}}{2 J+1}$ & 0 & $-2(J-1)$ \\
\hline \hline
\end{tabular}

Finally, we briefly compare our results to previous calculations. The only other shell model calculations we are aware of are those of Ref. [18] and Ref. [20]. The results of Ref. [20] are in agreement with ours while the preliminary results reported by Dubach et al. [18] appear to be very different. Their uncorrelated OPE-only rate for ${ }_{\Lambda}^{12} \mathrm{C}$ is listed as 3.4 which is about a factor of 2 larger than ours while adding initial SRC, FF, and FSI reduce this rate to 0.5. This amounts to a reduction factor of almost 7 , in contrast to our suppression of roughly a factor of 2 . Furthermore, their correlated rate for ${ }_{\Lambda}^{5} \mathrm{He}$ is listed as 0.9 , almost a factor of 2 larger than the ${ }_{\Lambda}^{12} \mathrm{C}$ result. Unfortunately, no details are given in Ref. [18] that address these problems. We note, however, that there are some unexplained inconsistencies between their recent results of Ref. [18] and what was reported ten years before in Ref. [50], where the correlated $\pi$-exchange decay rate for ${ }_{\Lambda}^{12} \mathrm{C}$ is 2.0 while the addition of the other mesons lowers this value to 1.2. In fact, these values are more consistent with their ${ }_{\Lambda}^{5} \mathrm{He}$ results, as well as with the effect of short-range correlations found in almost all studies of the nonmesonic weak decay either in nuclear matter or finite nuclei. Our results for $\Gamma_{n} / \Gamma_{p}$ again differ from what it is reported in Ref. [18], where a value $\Gamma_{n} / \Gamma_{p}=0.2$ is obtained for $\pi$ exchange alone but 0.83 when all the mesons are included. However, their results in finite nuclei are, surprisingly, quite different from their nuclear matter results, namely $\Gamma_{n} / \Gamma_{p}=0.06$ for $\pi$ exchange alone and 0.345 when all mesons are included. With regard to the asymmetry parameter, the nuclear matter results of Ref. [18] are qualitatively similar to our results for the Nijmegen couplings. They obtain a value $a_{\Lambda}=-0.192$ for $\pi$ exchange alone and -0.443 when all the mesons are considered.

\section{CONCLUSIONS}

In this study we have presented calculations for the weak nonmesonic decay mode $\Lambda N \rightarrow N N$ of $\Lambda$ hypernuclei. In contrast to most previous investigations performed in nuclear matter this work analyzed this hypernuclear decay in a nonrelativistic shell model approach. The initial hypernuclear and final nuclear structure are taken into account through spectroscopic factors. All possible initial and final relative orbital angular momenta are included in the baryon-baryon system. Realistic initial and final short-range correlations (SRC) obtained from $\Lambda N$ and $N N$ interactions based on the Nijmegen baryon-baryon potential are employed in order to treat the nuclear structure details with as few approximations and ambiguities as possible. Our calculations were per- formed in a one-boson-exchange model that includes not only the long-ranged pion but also contributions from the other pseudoscalar mesons, the $\eta$ and $K$, as well as the vector mesons $\rho, \omega$, and $K^{*}$. The weak baryon-baryon-meson vertices were obtained using $\mathrm{SU}(6)$ and soft meson theorems for the PV vertices and the pole model for the PC vertices. The primary goal of this work was to reduce nuclear structure uncertainties as much as possible so that our framework can be used to extract these weak baryon-baryon-meson couplings.

Total decay rates evaluated with the full weak OBE potential fall within $15 \%$ of the value obtained with pion exchange only and reproduce the experimental data. This is due to the interference between the contributions of the heavier mesons whose individual influence on the decay rate can be substantial. Including the kaon exchange alone reduces the total rate by almost $50 \%$, this reduction is compensated by adding the other mesons, specifically the $K^{*}$. In contrast to previous studies we found little influence from the $\rho$ meson even when we used a different model for the weak $\Lambda N \rho$ couplings, similarly the $\omega$ contributes at the $10 \%$ level. The dominant contribution beyond the pion-exchange mechanism is clearly the kaon exchange, followed by the $K^{*}$ which tends to partially cancel the effects of the kaon. It is therefore imperative that future studies include both strange mesons simultaneously. The importance of kaon exchange makes it possible to see the effects of modifying the weak NNK couplings by one-loop corrections to the leading order in $\chi \mathrm{PT}$. Including these loop graphs leads to a reduction of the $N N K$ couplings from their tree-level value up to $50 \%$, which in turns modifies the rates by up to $20 \%$. Future experiments should be able to verify this effect.

We found the dominance of strange mesons to be even more pronounced in the partial rates and their ratio. Including the kaon reduces this ratio by more than a factor of 3 , which again is compensated by the $K^{*}$. Furthermore, this ratio turns out to be sensitive to the choice of strongcoupling constants as well. Using the Nijmegen strong couplings reduces the ratio by $30 \%$ from its pion-only value while the use of the Jülich strong couplings leads to a change of only a few percent. This finding indicates the need for improved $Y N$ potentials with better determined strong couplings at the hyperon-nucleon-meson vertices. Both theoretical values are far away from the experimental data, even though the error bars are still large. It appears to be impossible to reconcile these discrepancies within a one-boson exchange potential. In order to approach the experimental values the weak couplings of the heavier mesons would have to 
be unreasonably large which would yield very large total rates incompatible with the data. If future experiments with improved partial rates confirm the present trend new mechanisms of a different kind would have to be introduced to resolve this puzzle. In contrast to the previous observables we found the proton asymmetry to be very sensitive to the $\rho$-exchange while the influence of the kaon is more moderate. This polarization observable is therefore an important addition to the set of observables since its sensitivities are different from the total and partial rates. The results of our calculations are within the very large current error bounds.

\section{A. Criticisms}

Our study clearly indicates that further theoretical effort must be invested to understand the dynamics of the nonmesonic weak hypernuclear decay. Within the one-meson exchange picture it would be desirable to use weak-coupling constants developed with more sophisticated approaches. A beginning has been made by Savage and Springer [46] in their evaluation of the weak $N N K$ couplings including oneloop corrections to the leading order in $\chi \mathrm{PT}$ and the effect has been found to be important. However, an understanding of the weak $\Lambda N \pi$ and $\Sigma N \pi$ couplings within the framework of chiral Lagrangians is still missing. Furthermore, due to the importance of the $K^{*}$ meson it would be desirable to recalculate its weak $N N K^{*}$ couplings in improved models as well. Several recent studies [21] have gone beyond the conventional picture of meson exchange and have developed mechanisms based purely on quark degrees of freedom. One should keep in mind, however, that such models have not always been able to reproduce the experimentally measured free hyperon decays.

Another avenue that is currently being pursued is the validity of the $\Delta I=1 / 2$ rule in the $\Lambda N \rightarrow N N$ process. While this empirical rule is well established for the free hyperon and kaon decays there is some indication that it could be violated for the $\Lambda N \rightarrow N N$ process. Within the framework of $\mathrm{SU}(3)$ and soft meson theorems the weak vertices of $N N K$ and $\Lambda N \eta$ are related to the observable $\Lambda N \pi$ decay, therefore, one would expect small $\Delta I=3 / 2$ contributions for these mesons. On the other hand, the vector meson vertices can receive substantial contributions from factorization terms which have been shown not to fulfil the $\Delta I=1 / 2$ rule [22]. The attractive feature of these additional terms is their strong influence on the ratio $\Gamma_{n} / \Gamma_{p}$.

On the level of implanting the basic $\Lambda N \rightarrow N N$ amplitude into the nucleus uncertainties have been minimized our study by treating each ingredient as well as possible. Nevertheless, within the framework of the impulse approximation and the shell model the short-range correlations, the spectroscopic factors and the single particle wave functions still come from separate sources. This dilemma can be avoided in rigorous few-body calculations with realistic wave functions. The nonmesonic decay of the hypertriton can be calculated using correlated three-body hypernuclear wave functions for the initial hypertriton state and continuum Faddeev solutions for the three-nucleon scattering state [61]. Thus, all nuclear structure input is generated from the same underlying $Y N$ and $N N$ potentials, eliminating the ambiguities of the shell model approach. It is therefore of utmost importance to pur- sue experimental measurements of the nonmesonic decay of the hypertriton.

\section{B. Outlook}

On the experimental side, it is critical to obtain new high accuracy data soon. Improved partial rates for the protonand neutron-induced decay modes are especially important. Of help would be to not only measure rates but also exclusive spectra of the decay products. Such distributions would be significant to disentagle the effects of the $\Lambda N N \rightarrow N N N$ process from the two-body process discussed here. Beyond improving the present data base for the weak decay of $\Lambda$ hypernuclei, there are two more avenues which would aid our understanding of the weak $\Delta S=1$ hadronic interaction.

First, with the advent of new, high precision proton accelerators such as COSY in Jülich, it may become possible to perform a direct study of the time-reversed process $p n \rightarrow \Lambda p$ [62]. While the very low cross sections present in this direct investigation of the $\Delta S=1$ baryon-baryon interaction will be difficult to measure, high efficiency detection schemes should allow determining a branching ratio of $10^{-13}$. Thus, the strangeness changing hadronic weak interaction could be studied similarly to the weak parity-violating $N N$ interaction. The asymmetry of this reaction has been measured at several kinematics which are sensitive to different parts of the meson exchange potential. Furthermore, measuring the $p n \rightarrow \Lambda p$ process directly would give access to a number of polarization observables since the $\Lambda$ is selfanalyzing.

Secondly, the hypernuclear weak decay studies should be extended to double- $\Lambda$ hypernuclei. Very few events involving these exotic objects - whose very existence would place stringent constraints on the existence of the elusive $H$ dibaryon - have been reported. Studying the weak decay of these objects would open the door to a number of new exotic $\Lambda$-induced decays: $\Lambda \Lambda \rightarrow \Lambda N$ and $\Lambda \Lambda \rightarrow \Sigma N$. Both of these decays would involve hyperons in the final state and should be distinguishable from the ordinary $\Lambda N \rightarrow N N$ mode. Especially the $\Lambda \Lambda \rightarrow \Lambda N$ channel would be intruiging since the dominant pion exchange is forbidden, thus this reaction would have to occur mostly through kaon exchange. One would therefore gain access to the $\Lambda \Lambda K$ vertex.

Even with the demise of KAON, the promising efforts at KEK with an improved measurement of the ${ }_{\Lambda}^{5} \mathrm{He}$ decay, the continuing program at BNL, and the advent of the hypernuclear physics program (FINUDA) [60] at DAФNE represent excellent opportunities to obtain new valuable information that will shed light onto the still unresolved problems of the weak decay of hypernuclei.

\section{ACKNOWLEDGMENTS}

We are grateful to Professor P. Pascual for clarifying discussions regarding sign conventions and relations between coupling constants. The work of C.B. was supported by US-DOE Grant No. DE-FG02-95-ER40907 while the work of A.P. and A.R. was supported by DGICYT Contract No. PB92-0761 (Spain) and by the Generalitat de Catalunya Grant No. GRQ94-1022. This work has received support from NATO Grant No. CRG 960132. A.P. acknowledges the Ministerio de Educación y Ciencia (Spain) for financial support. 


\section{APPENDIX: COEFFICIENTS $\left\langle\left(L^{\prime} S\right) J M_{J}\left|\hat{O}_{\alpha}\right|\left(L_{R} S_{0}\right) J M_{J}\right\rangle$}

Spin-Spin transition:

$$
\left\langle\left(L^{\prime} S\right) J M_{J}\left|\hat{O}_{\alpha}\right|\left(L_{r} S_{0}\right) J M_{J}\right\rangle=(2 S(S+1)-3) \delta_{L_{r} L^{\prime}} \delta_{S_{i}} \mathbf{S}
$$

Tensor transition:

$$
\left\langle\left(L^{\prime} S\right) J M_{J}\left|\hat{O}_{\alpha}\right|\left(L_{r} S_{0}\right) J M_{J}\right\rangle=S_{L_{r} L^{\prime}}^{J} \delta_{S_{0} S} \delta_{S 1}
$$

where the coefficients $S_{L_{r} L^{\prime}}^{J}$ are given in Table XI.

$P V$ transition. Pseudoscalar mesons:

$$
\left\langle\left(L^{\prime} S\right) J M_{J}\left|\hat{O}_{\alpha}\right|\left(L_{r} S_{0}\right) J M_{J}\right\rangle=(-1)^{J+1-L^{\prime}} \sqrt{6} \sqrt{2 S_{0}+1} \sqrt{2 L_{r}+1} \sqrt{2 S+1}\left\langle 10 L_{r} 0 \mid L^{\prime} 0\right\rangle\left(\begin{array}{ccc}
\frac{1}{2} & \frac{1}{2} & S_{0} \\
S & 1 & \frac{1}{2}
\end{array}\right)\left(\begin{array}{ccc}
L^{\prime} & L_{r} & 1 \\
S_{0} & S & J
\end{array}\right) .
$$

PV transition. Vector mesons:

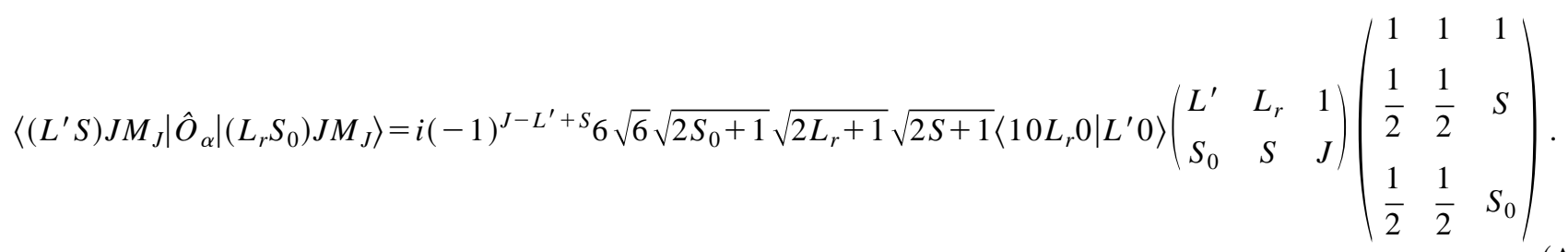

[1] H. Bandō, T. Motoba, and J. Žofka, Int. J. Mod. Phys. A 5, 4021 (1990).

[2] W. Cheston and H. Primakoff, Phys. Rev. 92, 1537 (1953).

[3] W. M. Alberico, A. De Pace, M. Ericson, and A. Molinari, Phys. Lett. B 256, 134 (1991).

[4] A. Ramos, E. Oset, and L. L. Salcedo, Phys. Rev. C 50, 2314 (1994).

[5] A. Ramos, E. Oset, and L. L. Salcedo, Few-Body Syst. Suppl. 9, 490 (1995).

[6] S. Polikanov, Nucl. Phys. A478, 805c (1988), and references therein.

[7] J. J. Szymanski et al., Phys. Rev. C 43, 849 (1991).

[8] R. A. Schumacher, in Proceedings of the U.S.-Japan Seminar on the Properties and Interactions of Hyperons, Maui, Hawaii, 1993, edited by B. F. Gibson, P. D. Barnes, and K. Nakai (World Scientific, Singapore, 1994), p. 85.

[9] H. Noumi et al., Phys. Rev. C 52, 2936 (1995).

[10] S. Ajimura et al., Phys. Lett. B 282, 293 (1992).

[11] J. Cohen, Progress in Particle and Nuclear Physics, edited by A. Faessler (Pergamon, New York, 1990), Vol. 25, p. 139.

[12] R. H. Dalitz and G. Rajasekharan, Phys. Lett. 1, 58 (1962); M. M. Block and R. H. Dalitz, Phys. Rev. Lett. 11, 96 (1963).

[13] J. B. Adams, Phys. Rev. 156, 1611 (1967).

[14] E. Oset and L. L. Salcedo, Nucl. Phys. A443, 704 (1985).

[15] B. H. J. McKellar and B. F. Gibson, Phys. Rev. C 30, 322 (1984).

[16] G. Nardulli, Phys. Rev. C 38, 32 (1988).

[17] J. F. Dubach, Nucl. Phys. A450, 71c (1986).

[18] J. F. Dubach, G. B. Feldman, B. R. Holstein, and L. de la Torre, Ann. Phys. (N.Y.) 249, 146 (1996).
[19] C. Bennhold and A. Ramos, Phys. Rev. C 45, 3017 (1992).

[20] C.-Y. Cheung, D. P. Heddle, and L. S. Kisslinger, Phys. Rev. C 27, 335 (1983); D. P. Heddle and L. S. Kisslinger, ibid. 33, 608 (1986).

[21] T. Inoue, S. Takeuchi, and M. Oka, Nucl. Phys. A597, 563 (1996).

[22] K. Maltman and M. Shmatikov, Phys. Lett. B 331, 1 (1994).

[23] K. Itonaga, T. Ueda, and T. Motoba, Nucl. Phys. A585, 331c (1995); Weak and Electromagnetic Interactions in Nuclei, edited by H. Ejiri, T. Kishimoto, and T. Sato (World Scientific, Singapore, 1995), p. 546.

[24] M. Shmatikov, Nucl. Phys. A580, 538 (1994).

[25] A. Ramos, C. Bennhold, E. van Meijgaard, and B. K. Jennings, Phys. Lett. B 264, 233 (1991).

[26] A. Ramos, E. van Meijgaard, C. Bennhold, and B. K. Jennings, Nucl. Phys. A544, 703 (1992).

[27] A. Parreño, A. Ramos, C. Bennhold, and D. Halderson, in Dynamical Features of Nuclei and Finite Fermi Systems, edited by X. Viñas, M. Pi, and A. Ramos (World Scientific, Singapore, 1994), p. 318.

[28] A. Parreño, A. Ramos, and E. Oset, Phys. Rev. C 51, 2477 (1995); A. Parreño, E. Oset, and A. Ramos, Few-Body Syst. Suppl. 9, 293 (1995).

[29] S. Cohen and D. Kurath, Nucl. Phys. A101, 1 (1967).

[30] C. Bennhold, Phys. Rev. C 43, 775 (1991).

[31] R. V. Reid, Ann. Phys. (N.Y.) 50, 411 (1968).

[32] V. G. Stoks, R. A. M. Klomp, C. P. F. Terheggen, and J. J. de Swart, Phys. Rev. C 49, 2950 (1994).

[33] M. I. Haftel and F. Tabakin, Nucl. Phys. A158, 1 (1970).

[34] A. Perreño, A. Ramos, and C. Bennhold, Phys. Rev. C 52, 
R1768 (1995); 54, 1500(E) (1996).

[35] C. Bennhold, A. Parreño, and A. Ramos, Few-Body Syst. Suppl. 9, 475 (1995).

[36] W. Weise, Nucl. Phys. A278, 402 (1977).

[37] D. Halderson, Phys. Rev. C 48, 581 (1993).

[38] M. N. Nagels, T. A. Rijken, and J. J. de Swart, Phys. Rev. D 15, 2547 (1977); P. M. M. Maessen, Th. A. Rijken, and J. J. de Swart, Phys. Rev. C 40, 2226 (1989).

[39] K. Takeuchi, H. Takaki, and H. Bandō, Prog. Theor. Phys. 73, 841 (1985).

[40] B. Holzenkamp, K. Holinde, and J. Speth, Nucl. Phys. A500, 485 (1989).

[41] J. D. Bjorken and S. D. Drell, Relativistic Quantum Mechanics (McGraw-Hill, New York, 1964).

[42] L. de la Torre, Ph.D. thesis, University of Massachusetts, 1982.

[43] A. P. Balanchandran et al., Phys. Rev. 153, 1553 (1967).

[44] J. F. Donoghue et al., Phys. Rep. 131, 319 (1986).

[45] K. Maltman and M. Shmatikov, Phys. Rev. C 51, 1576 (1995).

[46] M. J. Savage and R. P. Springer, Phys. Rev. C 53, 441 (1996); 54, 2786(E) (1996).

[47] B. F. Gibson, Nuovo Cimento A 102, 367 (1989).

[48] E. Jenkins, Nucl. Phys. B375, 561 (1992).

[49] L. Tiator, C. Bennhold, and S. S. Kamalov, Nucl. Phys. A580, 455 (1994).

[50] J.F. Dubach, in Weak and Electromagnetic Interactions in $\mathrm{Nu}$ clei, Heidelberg, 1986, edited by H. V. Klapdor (SpringerVerlag, Berlin, 1986), p. 576.
[51] A. Montwill et al., Nucl. Phys. A234, 413 (1974).

[52] A. Ramos, M. J. Vicente-Vacas, and E. Oset, Phys. Rev. C 55, 735 (1997).

[53] K. Itonaga, T. Motoba, O. Richter, and M. Sotona, Phys. Rev. C 49, 1045 (1994).

[54] D. J. Millener, in Proceedings of the LAMPF Workshop on $(\pi, K)$ Physics, AIP Conf. Proc. No. 224, edited by B. J. Gibson, W. R. Gibbs, and M. B. Johnson (AIP, New York, 1991), p. 185.

[55] H. Ejiri, T. Kishimoto, and H. Noumi, Phys. Lett. B 225, 35 (1989).

[56] E. H. Auerbach, A. J. Baltz, C. B. Dover, A. Gal, S. H. Kahana, L. Ludeking, and D. J. Millener, Ann. Phys. (N.Y.) 148, 381 (1983).

[57] R. H. Dalitz, D. H. Davies, and D. N. Tovee, Nucl. Phys. A450, 311c (1986).

[58] T. Kishimoto, Nucl. Phys. A585, 205c (1995).

[59] T. Motoba and K. Itonaga, Nucl. Phys. A577, 293c (1994).

[60] FINUDA Collaboration, A. Zenoni, spokesperson, Proceedings of the Second Workshop on Physics and Detectors for $D A \Phi N E$, edited by R. Baldini, F. Bossi, G. Capon, and G. Pancheri (Frascati Physics Series Vol. IV, 1995), p. 293.

[61] J. Golak, K. Miyagawa, H. Kamada, H. Witala, W. Glöckle, A. Parreño, A. Ramos, and C. Bennhold, Phys. Rev. C 55, 2196 (1997).

[62] J. Haidenbauer et al., Phys. Rev. C 52, 3496 (1995). 